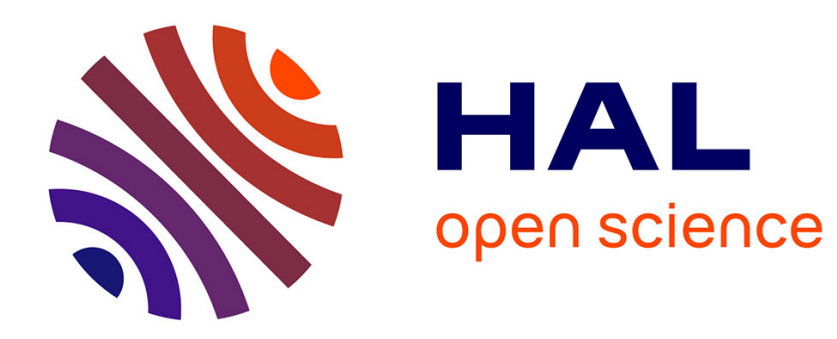

\title{
Linking Focusing and Resolution with Selection
}

Guillaume Burel

\section{To cite this version:}

Guillaume Burel. Linking Focusing and Resolution with Selection. ACM Transactions on Computational Logic, 2020, 21 (3), pp.1-30. 10.1145/3373276 . hal-02908808

\section{HAL Id: hal-02908808 \\ https://hal.science/hal-02908808}

Submitted on 29 Jul 2020

HAL is a multi-disciplinary open access archive for the deposit and dissemination of scientific research documents, whether they are published or not. The documents may come from teaching and research institutions in France or abroad, or from public or private research centers.
L'archive ouverte pluridisciplinaire HAL, est destinée au dépôt et à la diffusion de documents scientifiques de niveau recherche, publiés ou non, émanant des établissements d'enseignement et de recherche français ou étrangers, des laboratoires publics ou privés. 


\title{
Linking Focusing and Resolution with Selection
}

\author{
GUILLAUME BUREL, ENSIIE and Samovar, Télécom SudParis and CNRS, France and Inria and LSV, CNRS \\ and ENS Paris-Saclay, Université Paris-Saclay, France
}

Focusing and selection are techniques that shrink the proof-search space for respectively sequent calculi and resolution. To bring out a link between them, we generalize them both: we introduce a sequent calculus where each occurrence of an atomic formula can have a positive or a negative polarity; and a resolution method where each literal, whatever its sign, can be selected in input clauses. We prove the equivalence between cut-free proofs in this sequent calculus and derivations of the empty clause in that resolution method. Such a generalization is not semi-complete in general, which allows us to consider complete instances that correspond to theories of any logical strength. We present three complete instances: first, our framework allows us to show that ordinary focusing corresponds to hyperresolution and semantic resolution; the second instance is deduction modulo theory and the related framework called superdeduction; and a new setting, not captured by any existing framework, extends deduction modulo theory with rewriting rules having several left-hand sides, which restricts even more the proof-search space.

CCS Concepts: - Theory of computation $\rightarrow$ Proof theory; Automated reasoning.

Additional Key Words and Phrases: sequent calculus, refinements of resolution, deduction modulo theory, polarization

ACM Reference Format:

Guillaume Burel. 2019. Linking Focusing and Resolution with Selection. ACM Trans. Comput. Logic 1, 1, Article 1 (January 2019), 29 pages. https://doi.org/10.1145/nnnnnnn.nnnnnnn

\section{INTRODUCTION}

In addition to clever implementation techniques and data structures, a key point that explains the success of state-of-the-art automated theorem provers is the use of calculi that dramatically reduce proof-search space. In the last decades, the independent developments of two families of techniques can be highlighted. First, in the kind of methods based on resolution, proof-search space can be shrunk using ordering and selection techniques. The intuition is to restrict the application of the resolution rule to only some literals in a clause. If equality is considered, this leads to the superposition calculus [2] which is the base calculus of the currently most efficient automated provers for first-order classical logic. Second, in sequent calculi, Andreoli [1] introduced a technique called focusing to reduce non-determinism in the application of sequent-calculus rules. It works by first applying all invertible rules (those whose conclusion is logically equivalent to their premises) and second by chaining the application of non-invertible rules. Originally developed for linear logic, focusing has been extended to intuitionistic and classical first-order logic [31]. Focusing is mostly used in fields where sequent calculi, and related inverse and tableaux methods, are the

This article is an extended version of the paper presented at MFCS'18 [10]; it includes detailed proofs, and more examples and motivations.

Author's address: Guillaume Burel, guillaume.burel@ensiie.fr, ENSIIE and Samovar, Télécom SudParis and CNRS, 1, square de la Résistance, 91025, Évry, France, Inria and LSV, CNRS and ENS Paris-Saclay, Université Paris-Saclay, Cachan, France.

Permission to make digital or hard copies of all or part of this work for personal or classroom use is granted without fee provided that copies are not made or distributed for profit or commercial advantage and that copies bear this notice and the full citation on the first page. Copyrights for components of this work owned by others than the author(s) must be honored Abstracting with credit is permitted. To copy otherwise, or republish, to post on servers or to redistribute to lists, requires prior specific permission and/or a fee. Request permissions from permissions@acm.org.

(c) 2019 Copyright held by the owner/author(s). Publication rights licensed to ACM.

1529-3785/2019/1-ART1 \$15.00

https://doi.org/10.1145/nnnnnnn.nnnnnnn

ACM Trans. Comput. Logic, Vol. 1, No. 1, Article 1. Publication date: January 2019. 
most accurate proving methods. For instance, there exists tools for first-order linear logic [15], for intuitionistic logic [32] and for modal logic [33]. Focusing is also the key ingredient in Miller's ProofCert project aiming at building a universal framework for proof certification [18].

Despite their apparent lack of relation, we show in this paper that selection in refinements of the resolution calculus and focusing in sequent calculus are in fact strongly related, so that ordinary focusing in classical first-order logic corresponds actually to hyperresolution, where all negative literals are selected in a clause and are resolved at once. This connection is obtained by relaxing both techniques: concerning resolution, we allow any literal of the input clauses to be selected, whatever its sign; for the focusing part, we allow polarization not only of connectives, but also of all occurrences of literals. The main theorem of this paper, Theorem 4.2, shows that the sets of clauses whose insatisfiability can be proved by the resolution method with arbitrary input selection are exactly the sequents that have a cut-free proof in the generalized focusing setting.

This generalization allows us to cover a wider spectrum of proof systems. In particular, this permits to consider systems that search for proofs modulo some theory. Indeed, in real-world applications, proof obligations are often verified within one or several theories. This explains the interest in and the success of Satisfiability Modulo Theory tools in recent years. Embedding a theory in our framework amounts to giving an axiomatic presentation of it where some literals are selected.

By relaxing the conditions for selecting literals, our framework is not always refutationaly complete. However, this should not be considered as a drawback, but as an essential point to be able to represent efficiently all kinds of theories. Indeed, let us consider a proof-search method $\mathbb{P}(\mathcal{T})$ parameterized by a theory $\mathcal{T}$. Ideally, $\mathbb{P}(\mathcal{T})$ should be as efficient as a generic proof-search method if it is fed with a formula that is not related to the theory $\mathcal{T}$. In particular, if it tries to refute the true formula $T$, it should terminate, and with the answer "NO". Let us say that $\mathbb{P}(\mathcal{T})$ is relatively consistent if it is the case. As we pointed out with Dowek [11], we cannot have a generic proof of the completeness of a relatively consistent method $\mathbb{P}(\mathcal{T})$ that would work for all $\mathcal{T}$. Indeed, such a proof would imply the consistency of the theory $\mathcal{T}$, and, according to Gödel, this cannot be performed in $\mathcal{T}$ itself. So either the completeness of the proof system is proved once and for all, but it cannot represent theories that are logically at least as strong as that proof of completeness; or it is not complete in general but it can be proved to be complete for particular theories, whatever their logical strengths. What is interesting therefore is to give proofs of completeness of $\mathbb{P}(\mathcal{T})$ for particular theories $\mathcal{T}$.

Consequently, we give three instances of our framework, where we can have proofs of completeness. First, as stated above, we link ordinary focusing with hyperresolution, and, in the ground case, with semantic resolution. Second, we show that Deduction Modulo Theory [23] is also a particular instance of this framework, knowing that there exists numerous proof techniques to prove the completeness of Deduction Modulo a particular theory, for instance [9, 21, 24, 28]. Third, we show how completeness in our framework can be reduced to completeness of several instances of Deduction Modulo Theory, thus allowing to reuse these techniques.

To give an intuition about our framework, and to illustrate how much the proof-search space can be constrained without losing completeness, let us consider for example the theory defining the powerset:

$$
\forall X, \forall Y,(X \in \mathcal{P}(Y)) \Leftrightarrow(\forall Z,(Z \in X) \Rightarrow(Z \in Y))
$$


This theory can be put in clausal normal form, using $d$ as a Skolem symbol, and we select (by underlining them) some literals in these clauses ${ }^{1}$ :

$$
\begin{gathered}
\frac{\neg X \in \mathcal{P}(Y)}{} \vee \neg Z \in X \vee Z \in Y \\
\frac{X \in \mathcal{P}(Y)}{X \in \mathcal{P}(Y)} \vee \neg(X, Y) \in X \\
\underline{X(X, Y) \in Y}
\end{gathered}
$$

Using focusing in general, and in our framework in particular, the decomposition of connectives is so restricted that, given an axiom, a proof derivation decomposing this axiom would necessarily have only certain shapes. Thus, the axiom can be replaced by new inference rules, called synthetic rules, that are used instead of the derivation of those shapes. See Section 2.1, for more details. In our framework, this would lead to the following three synthetic rules, that can be used in place of the axioms (the explanation how these rules are obtained is given in Section 5.4):

$$
\begin{gathered}
(1) \vdash \frac{\Delta, u \in \mathcal{P}(v), t \in u, t \in v \vdash}{\Delta, u \in \mathcal{P}(v), t \in u \vdash} \quad(2) \vdash \frac{}{\Delta, \neg u \in \mathcal{P}(v), d(u, v) \in v \vdash} \\
(3) \vdash \frac{\Delta, \neg u \in \mathcal{P}(v), d(u, v) \in u \vdash}{\Delta, \neg u \in \mathcal{P}(v) \vdash}
\end{gathered}
$$

If one tries to prove transitivity of the membership in the powerset, i.e. to refute the formula $\exists A . \exists B . \exists C . A \in \mathcal{P}(B) \wedge B \in \mathcal{P}(C) \wedge \neg A \in \mathcal{P}(C)$, only one proof can be built, namely:

$$
\begin{aligned}
& \text { (2) } \\
& \text { (1) } \\
& \vdash \in \mathcal{P}(b), b \in \mathcal{P}(c), \neg a \in \mathcal{P}(c), d(a, c) \in a, d(a, c) \in b \vdash \\
& (1) \vdash \frac{a \in \mathcal{P}(b), b \in \mathcal{P}(c), \neg a \in \mathcal{P}(c), d(a, c) \in a \vdash}{\sim} \\
& \text { (3) } \\
& \wedge \vdash \overline{a \in \mathcal{P}(b) \wedge b \in \mathcal{P}(c) \wedge \neg a \in \mathcal{P}(c) \vdash} \\
& \exists \vdash \overline{\exists A . \exists B \cdot \exists C \cdot A \in \mathcal{P}(B) \wedge B \in \mathcal{P}(C) \wedge \neg A \in \mathcal{P}(C) \vdash}
\end{aligned}
$$

where the active formulas in a sequent are underwaved, and double lines indicate potentially several applications of an inference rule.

On the resolution side, clauses (1) to (3) lead to the following ground derived rules (see also Section 5.4):

$$
\begin{aligned}
& \begin{array}{ll}
\text { (1) } \frac{u \in \mathcal{P}(v) \vee C \quad t \in u \vee D}{t \in v \vee C \vee D} \quad \text { (2) } \frac{\neg u \in \mathcal{P}(v) \vee C}{d(u, v) \in u \vee C}
\end{array} \\
& \text { (3) } \frac{\neg u \in \mathcal{P}(v) \vee C \quad d(u, v) \in v \vee D}{C \vee D}
\end{aligned}
$$

Once again, there is only one proof of transitivity, i.e. starting from the set of clauses $\{a \in \mathcal{P}(b) ; b \in$ $\mathcal{P}(c) ; \neg a \in \mathcal{P}(c)\}$ :

\footnotetext{
${ }^{1}$ We use the associative-commutative-idempotent symbol $\vee$ in clauses to distinguish it from the symbol $\vee$ that is used in formulas.
} 


$$
\text { (3) } \frac{\neg a \in \mathcal{P}(c) \quad(1) \frac{b \in \mathcal{P}(c) \quad}{d(1) \frac{a \in \mathcal{P}(b) \quad(2) \frac{\neg a \in \mathcal{P}(c)}{d(a, c) \in a}}{d(a, c) \in b}}}{\square}
$$

and we cannot even infer other clauses than those. We let the reader compare with what happens if we use clauses (1) to (3) in resolution, even using the ordered resolution with selection refinement.

In the next section, we present the sequent calculus $\mathrm{LKF}^{\perp}$, which extends the calculus LKF of Liang and Miller [31]. Section 3 introduces the resolution method with arbitrary input selection. Then, the main theorem of Section 4, and of this paper, namely Theorem 4.2, establishes the link between the two proof systems. In Section 5 are highlighted particular instances where proofs of completeness can be obtained, which also permit to show an a priori unsuspected relation between existing proof systems such as for instance hyperresolution and ordinary focusing. We also show how to relate completeness in our setting to completeness in Deduction Modulo Theory.

\section{Related work}

As we do here, Goubault-Larrecq [27] proves completeness of several refinements of resolution, including hyperresolution and semantic resolution, using syntactic transformations instead of relying on the construction of a model.

Chaudhuri, Pfenning, and Price [16] show that hyperresolution for Horn clauses can be explained as an instance of a sequent calculus for intuitionistic linear logic with focusing where atomic formulas are given a negative polarity.

Farooque, Graham-Lengrand, and Mahboubi [26] developed a sequent calculus, based on focusing, that is able to simulate $\operatorname{DPLL}(\mathcal{T})$, the most common calculus used in SMT provers. The main difference with our framework is that in [26], the theory is considered as a black box which is called as an oracle. Here, the theory is considered as a first-class citizen.

Within the ProofCert project, resolution proofs can be checked by a kernel built upon a sequent calculus with focusing [18]. Based on this, the tool Checkers [17] is able to verify proofs coming from automated theorem provers based on resolution such as E-prover. Different from here, they translate resolution derivations using cuts to get smaller proofs.

Hermant [29] proves the correspondance between the cut-free fragment of a sequent calculus and a resolution method, in the setting of Deduction Modulo Theory. Since Deduction Modulo Theory is subsumed by our framework, Theorem 4.2 is a generalization of Hermant's work. Proving it is simpler in our setting because focusing restrains the shape of possible sequent calculus proofs, whereas Hermant had to prove technical lemmas to give proofs a canonical shape.

\section{Notations and conventions}

We use standard definitions for terms, predicates, formulas (with connectives $\perp, \top, \neg, \wedge, \vee$ and quantifiers $\forall, \exists$ ), sequents and substitutions. Since we consider classical logic, implication $A \Rightarrow B$ is syntactic sugar for $\neg A \vee B$, and similarly equivalence $A \Leftrightarrow B$ denotes $(A \Rightarrow B) \wedge(B \Rightarrow A)$. A literal is an atomic formula or its negation. A clause is a set of literals. We will identify a literal with the unit clause containing it. Unless stated otherwise, letters $P, Q, R, P^{\prime}, P_{1}, \ldots$ denote atomic formulas, $L, K, L^{\prime}, L_{1}, \ldots$ denote literals, $A, B, A^{\prime}, A_{1}, \ldots$ denote formulas, $C, D, C^{\prime}, C_{1}, \ldots$ denote clauses, $\Gamma, \Delta$ denote sets of clauses or multisets of formulas (depending on the context). In particular, sequents contain multisets of formulas. $A^{\perp}$ denotes the negation normal form of $\neg A$. 


\section{FOCUSING WITH POLARIZED OCCURRENCES OF ATOMIC FORMULAS}

Focusing was introduced by Andreoli [1] to restrict the non-determinism in some sequent calculus for linear logic. It relies on the alternation of two phases: During the asynchronous phase (sequents with $\Uparrow$ ), all invertible rules are applied on the formulas of the sequent. Recall that a rule is said invertible if its conclusion implies the conjunction of its premises. During the synchronous phase (sequents with $\Downarrow$ ), a particular formula is selected -the focus is on it- and all possible non-invertible rules are successively applied on it. This idea has been extended to intuitionistic and classical firstorder logic by Liang and Miller [31]. In these, connectives may have invertible and non-invertible versions of their sequent calculus rules. Therefore, one considers in that case two versions of a connective, one called positive when the right introduction rule is non-invertible, and one called negative when it is invertible. Some connectives, i.e. $\exists$ in classical logic, only have a positive version, and dually, others, such as $\forall$ in classical logic, only have a negative version. Given a usual formula, one can decide which version of a connective one wants to use at a particular occurrence, which is called a polarization of the formula. ${ }^{2}$ Note that the polarity of a connective does not affect its semantics, it only alters the shape of the sequent calculus proofs. Similarly, one can decide the polarity of each literal. If a literal with negative polarity $L$ is focused on in a branch, then this branch must necessarily be closed, with $L^{\perp}$ in the context. (See rule $\bar{\Downarrow}_{r}$ in Figure 1.)

In the ordinary presentation of focusing, this polarity is chosen globally for all occurrences of each atomic formula, and the polarity of $\neg P$ is defined as the inverse of that of $P$. In our setting, the polarity can be different for each occurrence of a literal. More precisely, the polarity is attached to the position of the literal in the formula. Let us consider the usual subformula relation: in particular, for all terms $t,\{t / x\} P$ is a subformula of $\forall x . P$ and of $\exists x . P$. In the same way as what happens for polarities of connectives in ordinary focusing, when considering subformulas in the premises of inference rules, the polarities of connectives and literals remain the same. In particular, if a literal $L$ has a negative polarity in e.g. $\forall x . P$, then $\{t / x\} L$ will have a negative polarity too in $\{t / x\} P$. Also, if formulas are copied by an inference rule (e.g. the formulas in $\Delta$ in the rule $\Downarrow V-$ of Figure 1), both copies in the premises contain the same polarities. Furthermore, when considering the application of a first-order term substitution to a formula, the polarities of the literals in the resulting formula remain the same. The polarity of a formula is defined as the polarity of its top connective.

Liang and Miller [31] introduce the sequent calculus LKF, and prove it to be complete for classical first-order logic. In Figure 1, we present the calculus $\mathrm{LKF}^{\perp}$, which is almost the same with the following differences:

- All formulas are put on the left-hand side of the sequent, instead of the right-hand side. Therefore, one does not try to prove a disjunction of formulas, but one tries to refute a conjunction of formulas. This is the same thanks to the dual nature of classical first-order logic, and this helps to be closer to the resolution derivations. Note that, consequently, the focus is on negative formulas, and invertible rules are applied on positive formulas.

- The polarity of atomic formulas is not chosen globally, but each occurrence of a literal can have a positive or a negative polarity. In particular, we can have two literals $L$ and $L^{\perp}$ which are both negative, or both positive. We denote by $\underline{L}$ the fact that the literal $L$ has a negative polarity. To be able to close branches on which we have two positive opposed literals, we add a rule $\pi r$. Note that in this rule, $L$ and $L^{\perp}$ are assumed to have a positive polarity.

We denote by $\Gamma \Uparrow \Delta \vdash$ (with $\Gamma$ or $\Delta$, possibly empty, containing polarized formulas) the fact that there exists a proof of the sequent $\Gamma \Uparrow \Delta \vdash$ in $\mathrm{LKF}^{\perp}$, that is, a derivation starting from this sequent

\footnotetext{
${ }^{2}$ Let us note that this notion of polarity is a standard denomination when dealing with focusing, and should not be confused with the more usual but unrelated notion defined by the parity of the negation-depth of a position in a formula.
} 
Asynchronous phase:

$$
\pi_{\vdash} \overline{\Gamma, L, L^{\perp} \Uparrow \vdash}
$$

$\Uparrow \exists \vdash \frac{\Gamma \Uparrow \Delta, A \vdash}{\Gamma \Uparrow \Delta, \exists x . A \vdash}(x$ not free in $\Gamma, \Delta)$

$\Uparrow \vee_{\vdash} \frac{\Gamma \Uparrow \Delta, A \vdash \quad \Gamma \Uparrow \Delta, B \vdash}{\Gamma \Uparrow \Delta, A \vee^{+} B \vdash}$

$$
\Uparrow \wedge \vdash \frac{\Gamma \Uparrow \Delta, A, B \vdash}{\Gamma \Uparrow \Delta, A \wedge^{+} B \vdash}
$$

$$
\Uparrow T r \frac{\Gamma \Uparrow \Delta r}{\Gamma \Uparrow \Delta, T r}
$$

Synchronous phase:

$$
\mathbb{\Downarrow}_{\vdash} \frac{\Gamma, L^{\perp} \Downarrow \underline{L} \vdash}{\Gamma \forall_{\vdash} \frac{\Gamma \Downarrow\{t / x\} A \vdash}{\Gamma \Downarrow \forall x . A \vdash}}
$$

$\Downarrow \perp \frac{}{\Gamma \Downarrow \perp \vdash}$

$\Downarrow \vee \vdash \frac{\Gamma \Downarrow A \vdash \quad \Gamma \Downarrow B \vdash}{\Gamma \Downarrow A \vee^{-} B \vdash}$

$$
\Downarrow \wedge_{1} \vdash \frac{\Gamma \Downarrow A \vdash}{\Gamma \Downarrow A \wedge^{-} B \vdash}
$$

$$
\Downarrow \wedge_{2} \vdash \frac{\Gamma \Downarrow B \vdash}{\Gamma \Downarrow A \wedge^{-} B \vdash}
$$
Focus $\frac{\Gamma, A \Downarrow A \vdash}{\Gamma, A \Uparrow \vdash}$
Release $\frac{\Gamma \Uparrow A \vdash}{\Gamma \Downarrow A \vdash}$
Store $\frac{\Gamma, A \Uparrow \Delta \mathbf{r}}{\Gamma \Uparrow A, \Delta \mathbf{r}}$
(A negative)
(A positive)
(A negative or literal)

Fig. 1. The sequent calculus $\mathrm{LKF}^{\perp}$

and whose branches are all closed (by $\widetilde{\Downarrow}_{\vdash}, \widehat{\pi}_{\vdash}$ or $\Downarrow \perp \vdash$ ). Thanks to focusing, such a proof has the following shape :

- Since one starts in an asynchronous $(\Uparrow)$ phase, invertible rules are successively applied to the positive formulas of $\Delta$, until one obtains negative formulas or literals that are put on the left of $\Uparrow$ using Store.

- When no formula appears on the right of $\Uparrow$, then either the branch is closed by $\pi_{r}-$; or the focus is put on a negative formula using Focus.

- In the latter case, one is now in synchronous $(\Downarrow)$ phase where non-invertible rules are successively applied to the formula upon which the focus is, until either the branch is closed using $\bar{\Downarrow}_{\vdash}$ or $\Downarrow_{\llcorner}$; or one obtains a positive formula and the synchronous phase ends using Release.

- In the latter case, one starts again in the asynchronous phase.

Focusing therefore strongly constrains the shape of possible proofs, and therefore reduces the proof-search space. The rule $\mathbb{\Downarrow}_{\mathfrak{r}}$ in particular imposes to close branches immediately when the focus is on a negative literal, and thus rules out many derivations.

Soundness of $\mathrm{LKF}^{\perp}$ comes directly from the soundness of the standard sequent calculus. Indeed, by replacing $\Uparrow$ and $\Downarrow$ by , (comma), one obtains rules that are admissible in the standard sequent calculus.

Example 2.1. Consider the polarized formula $\forall x .\left(P(x) \wedge^{-} \exists y . \neg P(y)\right)$ (the negation of the drinker paradox). The simplest refutation in $\mathrm{LKF}^{\perp}$ is : 


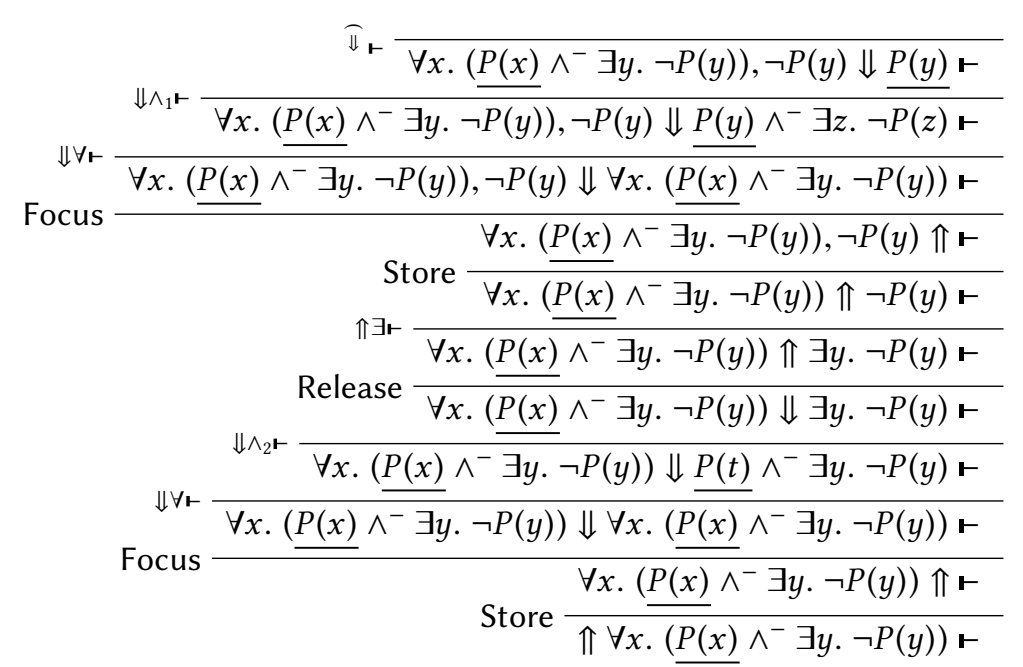

Note that it would not have been possible to apply $\Downarrow \wedge_{1} \vdash$ where $\Downarrow \wedge_{2} \vdash$ has been applied, because we would be stuck with the sequent $\forall x .\left(P(x) \wedge^{-} \exists y . \neg P(y)\right) \Downarrow P(t) \vdash$ that cannot be closed by $\mathbb{\Downarrow}_{\vdash}$.

To switch the polarity of a formula, e.g. to impose a change of phase, one can prefix it by so-called delays: $\delta^{-} A$ is negative whatever the polarity of $A$, whereas $\delta^{+} A$ is always positive. If a delay appears in the corresponding phase, then it can be silently ignored. For instance, one can have the following derivation:

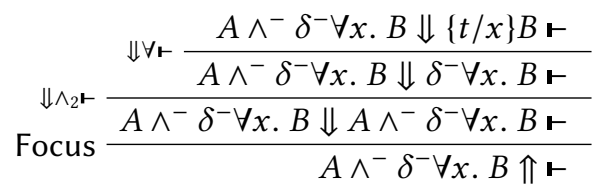

However, if the delay appears in the opposite phase, then the phase has to end. For instance, this gives the following derivation:

$$
\underset{\Downarrow \wedge_{2} \vdash}{\text { Release } \frac{\text { Store } \frac{A \wedge^{-} \delta^{+} \forall x . B, \forall x . B \Uparrow \vdash}{A \wedge^{-} \delta^{+} \forall x . B \Uparrow \forall x . B \vdash}}{A \wedge^{-} \delta^{+} \forall x . B \Downarrow \delta^{+} \forall x . B \vdash}}
$$

There is no need to extend the syntax of first-order formulas to include delays, because they can be defined for instance using quantifiers: $\delta^{-} A=\forall x$. $A$ and $\delta^{+} A=\exists x$. $A$, where $x$ is not free in $A$. We let the reader check that this induces indeed the desired behavior.

Note that proofs can be closed when the polarities of an atomic formula and its negation are both positive (rule $\tilde{\pi}_{\boldsymbol{r}}$ ), or when one is positive and the other negative (rule $\bar{\Downarrow}_{\boldsymbol{r}}$ ), but not when they are both negative. Therefore, this restricts how formulas that contains literals with negative polarities can interact one with the others, and this is the main point of $\mathrm{LKF}^{\perp}$ to reduce the proof-search space.

The sequent calculus $\mathrm{LKF}^{\perp}$ is not complete in general. One of the simplest examples of incompleteness is the sequent $\underline{P} \vee^{-} Q, \neg \underline{P} \vee^{-} Q, \neg Q \Uparrow \vdash$ which has no proof although $P \vee Q, \neg P \vee Q, \neg Q$ is not satisfiable.

One could choose a global polarization for atomic formulas and use delays to enforce the polarity of occurrences of literals without compromising completeness. However, this would break synchronization phases, and the proof-search space would not be as restricted as in our calculus. 
For instance, let $A$ be an arbitrary large tautology, and let $P$ be an atomic formula not appearing in $A$. Obviously, the set of formulas $P \vee A, \neg P$ is satisfiable and thus cannot be refuted. If one considers the polarization where both $P$ and $\neg P$ are negative in the formulas, one can quickly conclude that no derivation of $\underline{P} \vee^{-} A, \neg P \Uparrow \vdash$ exists. However, if one considers a global polarization where for instance $P$ is positive, even if $P$ is enforced to be negative in the first formula using a delay $\delta^{-}$, then a proof search can begin with

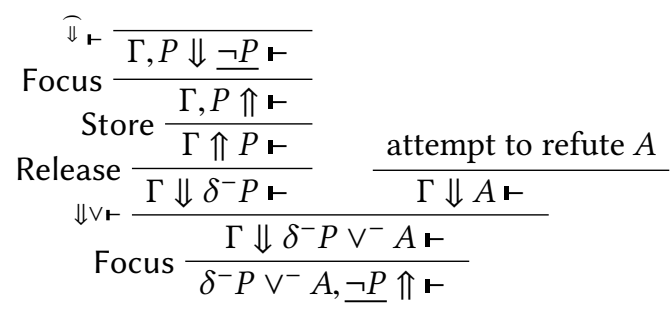

where $\Gamma$ is $\delta^{-} P \vee^{-} A, \neg P$. Depending on $A$, we cannot even guarantee that the attempt to refute $A$ will eventually end with a failure. Given a particular theory, it is therefore possible to carefully choose the polarization of the axioms of the theory so that the proof-search space is dramatically reduced but completeness w.r.t. that theory is not lost. Finding this particular polarization is the interesting part of our framework, since in general completeness can be lost. Section 5 presents some approaches to do this.

\subsection{Synthetic rules}

Restricting proof search using focusing leads to what are called synthetic rules (see for instance [16, pp.148-150] where they are called derived rules). The idea is to replace some formula $A$ in the context of the sequent by new inference rules. Instead of proving the sequent $A, \Gamma \Uparrow \Delta \vdash$ in $\mathrm{LKF}^{\perp}$, one proves $\Gamma \Uparrow \Delta \vdash$ in $\left(\mathrm{LKF}^{\perp}+\right.$ the synthetic rules obtained from $\left.A\right)$. Indeed, a proof focusing on $A$ can only have certain shapes, and thus instead of having $A$ in the context, it can be replaced by new rules synthesizing those shapes. For instance, the formula $\underline{P} \vee^{-}\left(Q \wedge^{-} \underline{R}\right)$ in a context $\Gamma$ can only lead to the following derivations when the focus is put on it:

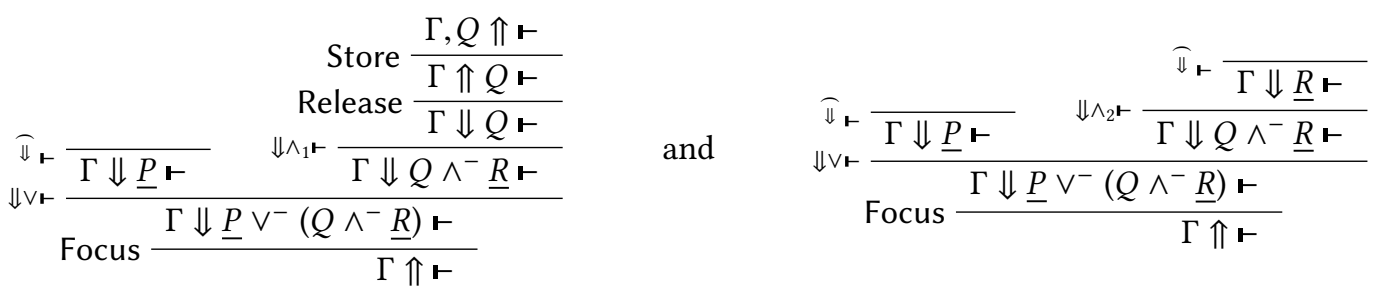

In the left derivation, $P^{\perp}$ must be in $\Gamma$ to be able to close the left branch, so $\Gamma$ is in fact of the form $\underline{P} \vee^{-}\left(Q \wedge^{-} \underline{R}\right), \Delta, P^{\perp}$. In the right one, $\Gamma$ must be of the form $\underline{P} \vee^{-}\left(Q \wedge^{-} \underline{R}\right), \Delta, P^{\perp}, R^{\perp}$. Instead of searching for a proof with $\underline{P} \vee^{-}\left(Q \wedge^{-} \underline{R}\right)$ in the context, the following two synthetic rules can therefore be used:

$$
\operatorname{Syn} 1 \frac{\Delta, P^{\perp}, Q \Uparrow \vdash}{\Delta, P^{\perp} \Uparrow \vdash} \quad \text { Syn } 2 \frac{}{\Delta, P^{\perp}, R^{\perp} \Uparrow \vdash}
$$

Note that in Syn 1 and Syn2, $\Delta$ is any context. In particular it need not contain $\underline{P} \vee^{-}\left(Q \wedge^{-} \underline{R}\right)$. This was the point of using synthetic rules instead of having $\underline{P} \vee^{-}\left(Q \wedge^{-} \underline{R}\right)$ in the context. This is completely equivalent in terms of provability because each application of a synthetic rule can be replaced by applying Focus on $\underline{P} \vee^{-}\left(Q \wedge^{-} \underline{R}\right)$ and following the derivation leading to the synthetic rule, and vice versa. This is used for instance in provers based on the inverse method and focusing [32]. 
For another example, this time with quantifiers, consider the formula $\forall x \cdot P(x) \vee^{-}\left((\exists y \cdot Q(x, y)) \wedge^{-}\right.$ $R(f(x)))$ in a context $\Gamma$. If the focus is put on this formula, only the following derivations are possible:

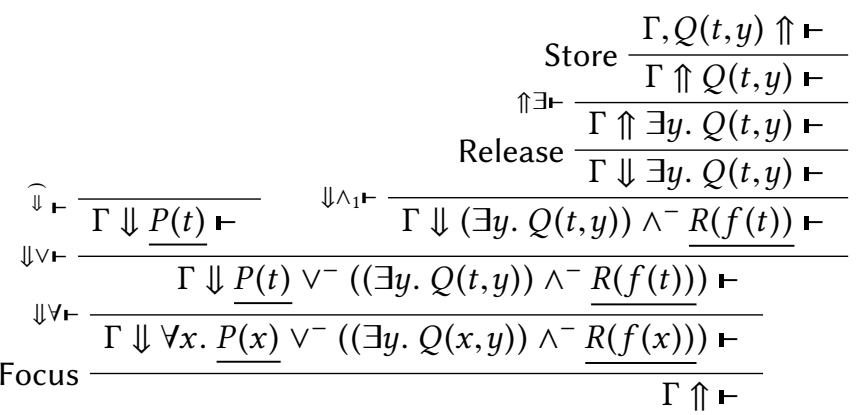

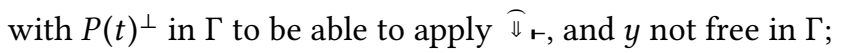

$$
\begin{aligned}
& \text { 『レ } \frac{}{\Gamma \Downarrow \underline{R(f(t))} \vdash} \\
& \underset{\Downarrow \vee}{\mathbb{\Downarrow}_{\vdash}} \frac{\overline{\Gamma \Downarrow P(t) \vdash}}{\Gamma \Downarrow P(t) \vee^{-}\left((\exists y \cdot Q(t, y)) \wedge^{-} R(f(t))\right) \vdash}
\end{aligned}
$$

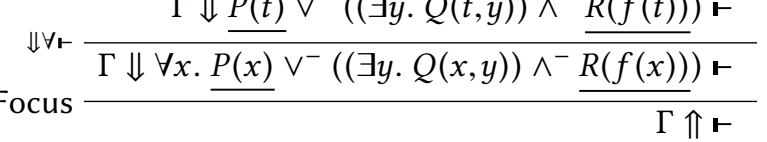

with both $P(t)^{\perp}$ and $R(f(t))^{\perp}$ in $\Gamma$ to be able to apply $\mathbb{\Downarrow}_{\vdash}$.

In the first case, $\Gamma$ is therefore $\forall x . P(x) \vee^{-}\left((\exists y . Q(x, y)) \wedge^{-} R(f(x))\right), \Delta, P(t)^{\perp}$; in the second

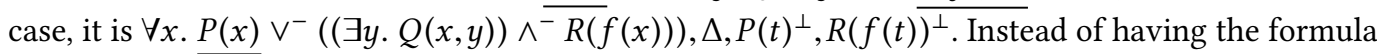
in the context, the following two synthetic rules can therefore be used:

Syn $1, \frac{\Delta, P(t)^{\perp}, Q(t, y) \Uparrow \vdash}{\Delta, P(t)^{\perp} \Uparrow \vdash} y$ not free in $\Delta, P(t)^{\perp}$

$$
\text { Syn2' } \overline{\Delta, P(t)^{\perp}, R(f(t))^{\perp} \Uparrow \vdash} .
$$

\section{RESOLUTION WITH INPUT SELECTION}

Two approaches can be used to reduce the proof-search space of the resolution calculus: first, one can restrict on which pairs of clauses the resolution rule can be applied; this leads for instance to the set-of-support strategy [37], in which clauses are split into two sets, called the theory and the set of support; at least one of the clauses involved in a resolution step must be in the set of support. Second, one can restrict which literals in the clauses can be resolved upon; those literals are said to be selected in the clause. Resolution with free selection is complete for Horn clauses, but incomplete in general. Selecting a subset of the negative literals (if no literal is selected, then any literal of the clause can be used in resolution) is however complete, and combining this with an ordering restriction on clauses with no selected literals leads to Ordered Resolution with Selection, which was introduced by Bachmair and Ganzinger [2] (see also Bachmair and Ganzinger [3]) as a complete refinement of resolution.

Resolution with Input Selection combines these two approaches. We will consider pairs composed of a clause and a subset of its literals (which can be empty). This subset of literals is the set of selected literals in the clause. We represent such a pair by underlining the selected literals in the clause. We will see that they indeed correspond to the literals that have a negative polarization in $\mathrm{LKF}^{\perp}$. We denote by $\mathcal{S}$ the second projection on such a pair. If at least one literal is selected in a clause, then only the selected literals can be used in Resolution. For generated clauses, we impose that $\mathcal{S}(C)=\emptyset$. Note that we can have several times the same clause with different selected literals. The inference rules of Resolution with Input Selection are presented in Fig. 2. As usual, variables 
Resolution $\frac{L \vee C \quad L^{\prime \perp} \vee D}{\sigma(C \vee D)}$
- $\mathcal{S}(L \vee C)=\emptyset$
- $\mathcal{S}\left(L^{\prime \perp} \vee D\right)=\emptyset$
- $\sigma$ is the most general unifier of $L={ }^{?} L^{\prime}$
Factoring $\frac{L \vee L^{\prime} \vee C}{\sigma(L \vee C)}$

- $\mathcal{S}\left(L \vee L^{\prime} \vee C\right)=\emptyset$

- $\sigma$ is the most general unifier of $L={ }^{?} L^{\prime}$

Resolution with Selection $\frac{K_{1} \vee \ldots \vee \underline{K_{n}} \vee C \quad K_{1}^{\prime \perp} \vee D_{1} \quad \ldots \quad K_{n}^{\prime \perp} \vee D_{n}}{\sigma\left(C \vee D_{1} \vee \ldots \vee D_{n}\right)}$

- $\mathcal{S}\left(K_{1} \vee \ldots \vee K_{n} \vee C\right)=\left\{K_{1} ; \ldots ; K_{n}\right\}$

- $\mathcal{S}\left(\overline{K_{i}^{\prime}}{ }^{\perp} \vee D_{i}\right) \overline{=\emptyset}$

- $\sigma$ is the mgu of the simultaneous unification problem $K_{1}=? K_{1}^{\prime}, \ldots, K_{n}={ }^{?} K_{n}^{\prime}$

Fig. 2. Resolution with Input Selection

are renamed in the clauses to avoid that premises of the inference rules share variables. We have two flavors of the resolution rule: the usual binary resolution, that is applied on two premises that do not select any literal; and Resolution with Selection that is applied on a clause in which $n$ literals are selected and $n$ clauses in which no literal is selected. Consequently, a clause with a non-empty selection cannot be resolved with a clause with a non-empty selection. By considering them as the theory part, and the clauses with an empty selection as the set of support, it is easy to see that Resolution with Input Selection is a generalization of the set-of-support strategy. Notwithstanding, note that neither Resolution with Input Selection is a generalization of Ordered Resolution with Selection nor the converse.

There are two ways to see resolution proofs: either as the successive derivations of new clauses until the empty clause is obtained, or as a tree whose root is the empty clause and whose leaves are included in the set of input clauses.

Definition 3.1 (Resolution derivation). We write $\Gamma \leadsto C$ if $C$ can be derived from some renaming of the clauses in $\Gamma$ using the inference rules Resolution with Selection, Resolution, or Factoring presented in Figure 2. We write $\Gamma \sim^{*} C$ if

- $C \in \Gamma$ or if

- there exists $D$ such that $\Gamma \leadsto D$ and $\Gamma, D \sim^{*} C$.

Definition 3.2 (Resolution tree). A resolution tree is a tree whose nodes contain clauses, such that $C$ is the father of $C_{1}, \ldots, C_{n}$ only if $C$ can be obtained from a renaming of $C_{1}, \ldots, C_{n}$ using one of the inference rules Resolution with Selection, Resolution, or Factoring. The multiset of the leaves of the tree is called the axioms of the tree. Its root is called the conclusion of the tree.

Of course, the two views are the same:

LEMMA 3.3. $\Gamma \sim^{*} C$ if and only if there exists a resolution tree whose axioms are included (as a set) in $\Gamma$, and whose conclusion is $C$.

Proof. For the "only if" direction, we construct the resolution tree by induction on the derivation length. If $C$ is in $\Gamma$, then we consider the tree with only one node containing $C$. The conclusion of the tree is therefore $C$, and its axioms are the multiset $\{C\}$ which is included as a set in $\Gamma$.

For the inductive case, suppose that there exists $D$ such that $\Gamma \leadsto D$ and $\Gamma, D \sim^{*} C$. By definition of $\leadsto$, there exists $C_{1}, \ldots, C_{n}$ in $\Gamma$ such that $D$ is obtained from a renaming of $C_{1}, \ldots, C_{n}$ using one of the inference rules Resolution with Selection, Resolution, or Factoring. By induction hypothesis, there exists a resolution tree whose conclusion is $C$ and whose axioms are included as a set in 
$\Gamma \cup\{D\}$. We can replace each occurrences of the axiom $D$ in the resolution tree by the subtree

\begin{tabular}{ccc}
$C_{1}$ & $\ldots$ & $C_{n}$ \\
\hline & &
\end{tabular} . The resulting tree is indeed a valid resolution tree, and its conclusion is $C$. Since all $C_{i}$ are in $\Gamma$, the axioms of this resolution tree are included, as a set, in $\Gamma$.

For the "if" direction, we proceed by induction on the size of the resolution tree (number of nodes). If the size is 1 , this means that the multiset of axioms is $\{C\}$. By assumption, this multiset is included as a set in $\Gamma$, hence $C \in \Gamma$. By definition of $\sim^{*}$, we have $\Gamma \sim^{*} C$.

For the inductive case, suppose that the size is $n>1$. Let us choose any node of the tree, containing a clause $D$, of which all children are leaves, containing respectively clauses $C_{1}, \ldots, C_{n}$. $D$ is therefore derived from a renaming of $C_{1}, \ldots, C_{n}$ using one of the inference rules Resolution with Selection, Resolution, or Factoring. By assumption, all $C_{i}$ belong to $\Gamma$. Therefore, by definition of $\leadsto$, we have

$$
\Gamma \leadsto D \text {. }
$$

Let us consider the resolution tree where we prune the subtree above $D$. Its size is strictly smaller than $n$, its conclusion is $C$ and its axioms are included, as a set, in $\Gamma \cup\{D\}$. By induction hypothesis, we have $\Gamma, D \sim^{*} C$. Consequently, with (4), $\Gamma \sim^{*} C$.

Note that transforming a derivation into a tree and back into a derivation is not the identity, because the derivation corresponds more precisely to a directed acyclic graph that is expanded into a tree by the transformation.

As usual in resolution methods, the goal is to produce the empty clause $\square$ starting from a set of clauses $\Gamma$ to show, since all rules are sound, that $\Gamma$ is unsatisfiable. Soundness of Resolution with Input Selection can be shown by remarking that the conclusion of each inference rule is a logical consequence of its premises, as in standard resolution.

Here again, the calculus is not complete in general: from the set of clauses $\underline{P} \vee Q, \neg P \vee Q, \neg Q$, no inference rule can be applied: to apply Resolution with Selection, we would need a clause where $P$, or $\neg P$, is not selected, and Resolution needs two clauses without selection.

\section{$4 \quad$ LKF $^{\perp}$ SIMULATES RESOLUTION WITH INPUT SELECTION}

To link $\mathrm{LKF}^{\perp}$ with Resolution with Input Selection, we need to indicate how clauses are related to polarized formulas.

Definition 4.1. Given a clause $C=\underline{L_{1}} \vee \cdots \vee L_{n} \vee K_{1} \vee \ldots \vee K_{m}$ whose free variables are $x_{1}, \ldots, x_{l}$ and such that $\mathcal{S}(C)=\left\{L_{1} ; \ldots ; \overline{L_{n}}\right\}$, we define the associated formula $\ulcorner C\urcorner$ to be $\forall x_{1}, \ldots, x_{l} . L_{1} \vee^{-} \ldots \vee^{-} L_{n} \vee^{-} \delta^{-}\left(K_{1} \vee^{+} \ldots \vee^{+} K_{m}\right) .\ulcorner C\urcorner$ is said to be clausal. By extension, $\ulcorner\Gamma\urcorner$ is the set of the formulas associated to the clauses of the set $\Gamma$.

Note that the formula associated to the renaming of a clause is the same (modulo $\alpha$-equivalence) as the formula associated to the clause.

Note that if $\mathcal{S}(C) \neq \emptyset$, or if $C$ is not ground, then the negative delay is useless. Indeed, in such cases, one is already in the synchronous phase when arriving at the subformula at that position, because the surrounding connective is either $\forall$ or $\mathrm{V}^{-}$. Adding the delay makes the translation of a clause always negative, even in the case of a ground clause without selection. It makes the statement and the proof of Theorem 4.7 more simple.

The main theorem of this article relates $\mathrm{LKF}^{\perp}$ with Resolution with Input Selection:

THeOREM 4.2. Let $\Gamma$ be a set of clauses. We have $\ulcorner\Gamma\urcorner \Uparrow \vdash$ iff $\Gamma \sim^{*} \square$.

The proof is detailed in the following subsections. 


\subsection{From Focused Proofs to Resolution Derivations}

We need a few lemmas to prove the first direction. Note that these lemmas work on clauses where the selection is empty, which is why we have the restriction that generated clauses do not select literals in Resolution with Input Selection.

Lemma 4.3. For all sets of clauses $\Gamma$, for all clauses $C_{1}, \ldots, C_{n}$ and $D$ such that $\mathcal{S}\left(C_{i}\right)=\emptyset$ for all $i$ and $\mathcal{S}(D)=\emptyset$, if $\Gamma, C_{1}, \ldots, C_{n} \sim^{*} \square$ and $\Gamma, D \sim^{*} \square$, then $\Gamma, C_{1} \vee D, \ldots, C_{n} \vee D \sim^{*} \square$ where $\mathcal{S}\left(C_{i} \vee D\right)=\emptyset$ for all $i$.

Proof. By induction on the derivation length of $\Gamma, C_{1}, \ldots, C_{n} \sim^{*} \square$, generalizing on $\Gamma$.

The base case is when $\square \in \Gamma$ or there exists $i$ such that $\square=C_{i}$. In the former case, trivially $\Gamma, C_{1} \vee D, \ldots, C_{n} \vee D \sim * \square$. In the latter case, $C_{i} \vee D=D$, and since $\Gamma, D \sim^{*} \square$ we have a fortiori $\Gamma, C_{1} \vee D, \ldots, C_{n} \vee D \sim *$ ๑.

For the inductive case, suppose that there exists $C_{n+1}$ such that $\Gamma, C_{1}, \ldots, C_{n} \leadsto C_{n+1}$ and $\Gamma, C_{1}, \ldots, C_{n}, C_{n+1} \sim^{*} \square$.

There are two cases:

- $C_{n+1}$ is derived using other clauses than one of the $C_{i}$. We therefore have $\Gamma, C_{1} \vee D, \ldots, C_{n} \vee D \sim C_{n+1}$.

We can apply the induction hypothesis on $\Gamma, C_{1}, \ldots, C_{n}, C_{n+1} \sim{ }^{*} \square$, which can be viewed as $\Gamma, C_{n+1}, C_{1}, \ldots, C_{n} \sim^{*} \square$. Indeed, by weakening we have $\Gamma, C_{n+1}, D \sim^{*} \square$. We obtain $\Gamma, C_{n+1}, C_{1} \vee D, \ldots, C_{n} \vee D \sim^{*} \square$. By definition of $\sim^{*}$ we therefore have $\Gamma, C_{1} \vee D, \ldots, C_{n} \vee D \sim * \square$.

- At least one of the parents of $C_{n+1}$ is some $C_{i}$. Since no literal is selected in the $C_{i}$, they can only be side clauses of Resolution with Selection, or any clause in Resolution or Factoring. We can therefore derive $C_{n+1} \vee D$ from $\Gamma, C_{1} \vee D, \ldots, C_{n} \vee D$ with the same inference rule that produced $C_{n+1}$. We can apply the induction hypothesis on $\Gamma, C_{1}, \ldots, C_{n}, C_{n+1} \sim^{*}$ 口 which gives us $\Gamma, C_{1} \vee D, \ldots, C_{n} \vee D, C_{n+1} \vee D \sim^{*} \square$.

Hence $\Gamma, C_{1} \vee D, \ldots, C_{n} \vee D \leadsto * \square$.

Corollary 4.4. For all sets of clauses $\Gamma$, for all clauses $C_{1}, \ldots, C_{n}$ such that $\mathcal{S}\left(C_{i}\right)=\emptyset$ for all $i$, if $\Gamma, C_{i} \sim^{*} \square$ for all $i$ then $\Gamma, C_{1} \vee \cdots \vee C_{n} \sim^{*} \square$ where $\mathcal{S}\left(C_{1} \vee \cdots \vee C_{n}\right)=\emptyset$.

LEMMA 4.5. For all multisets of clauses $\Gamma$, for all substitutions $\theta$, for all clauses $C$, for all resolution trees whose axioms are the multiset $\{\theta C\} \cup \Gamma$ with $\mathcal{S}(\theta C)=\emptyset$, and whose conclusion is $\square$, there exists a resolution tree whose axioms are the multiset $\{C\} \cup \Gamma$ with $\mathcal{S}(C)=\emptyset$, and whose conclusion is $\square$.

Proof. By induction on the lexicographic combination of the size (number of nodes) of the tree and the cardinal of $C$.

If the tree size is 1 , this means that $\Gamma=\emptyset$ and $\theta C=\square$. Thus, $C=\square$ too and we have a trivial tree of size 1 whose conclusion is $\square$ and whose multiset of axioms is $\{\square\}$.

If the tree size is $n>1$, let us consider the inference rule of which $\theta C$ is a premise.

If it is Factoring, then the conclusion of the inference rule is $\sigma \theta C$, where $\sigma$ is the most general unifier of two literals of $\theta C$. Let us consider the tree where we prune Factoring $\frac{\theta C}{\sigma \theta C}$ to have just $\sigma \theta C$ as an axiom. The axioms of this tree are $\{\sigma \theta C\} \cup \Gamma$ and its conclusion is $\square$. Its size is $n-1$. By induction hypothesis, using $\sigma \theta$ instead of $\theta$, we obtain a resolution tree whose axioms are $\{C\} \cup \Gamma$ and whose conclusion is $\square$, as was expected.

Let us consider the case when the inference rule is Resolution with Selection or Resolution. Since $\mathcal{S}(\theta C)=\emptyset$, only one literal of $\theta C$ is used in the inference rule. Let it be denoted by $L$, then $\theta C$ is $L \vee \theta C^{\prime}$, and $C$ is $L_{1} \vee \ldots \vee L_{m} \vee C^{\prime}$ where the $L_{i}$ are exactly the literals of $C$ such that

$$
\theta L_{i}=L
$$


If $m>1$, since $\theta L_{1}=\theta L_{2}=L$, the literals $L_{1}$ and $L_{2}$ can be unified. Let $\omega$ be their most general unifier. By definition, there exists $\theta^{\prime}$ such that $\theta=\theta^{\prime} \omega$. The axioms of the resolution tree are therefore the multiset $\left\{\theta^{\prime} \omega C\right\} \cup \Gamma$. The cardinal of $\omega C$ is smaller than the cardinal of $C$, since at least $\omega L_{1}$ and $\omega L_{2}$ are identical. By induction hypothesis, using $\theta^{\prime}$ instead of $\theta$, we get a resolution tree whose axioms are $\{\omega C\} \cup \Gamma$ and whose conclusion is $\square$. We can extend this resolution tree by replacing $\omega C$ with the subtree Factoring $\frac{C}{\omega C}$, thus obtaining a resolution tree whose axioms are $\{C\} \cup \Gamma$ and whose conclusion is $\square$, as was expected.

If $m=1$, since $\theta C=L \vee \theta C^{\prime}$ where $L$ is the literal used in the inference rule, the latter is therefore of the form $\frac{\theta C \quad C_{1} \vee D_{1} \quad \ldots \quad C_{k} \vee D_{k}}{\sigma\left(\theta C^{\prime} \vee D_{1} \vee \ldots D_{k}\right)}$ where $\sigma$ is the most general solution of a unification problem of the form $L={ }^{?} L^{\prime}$, Prob for some set of equations Prob (which may be non-empty in the Resolution with Selection case). In particular, this means that

$$
\sigma L=\sigma L^{\prime} .
$$

Since clauses are renamed before applying inference rules, we can assume that the variables of all $C_{i} \vee D_{i}$ are not in the domain of $\theta$. In particular, $L^{\prime}$ and all literals in Prob are not affected by $\theta$. Therefore,

$$
L^{\prime}=\theta L^{\prime}
$$

and

$$
D_{i}=\theta D_{i} \quad \text { for all } 1 \leq i \leq k .
$$

Combining (5), (6), and (7), we obtain

$$
\sigma \theta L_{1}=\sigma \theta L^{\prime}
$$

$\sigma \theta$ is therefore a solution of the unification problem $L_{1}=? L^{\prime}$, Prob (recall that variables in Prob are unaffected by $\theta$ ). Let $\mu$ be its most general solution. By definition, there exists $\kappa$ such that

$$
\sigma \theta=\kappa \mu \text {. }
$$

The conclusion of the inference rule is

$$
\begin{aligned}
& \sigma\left(\theta C^{\prime} \vee D_{1} \vee \ldots D_{k}\right) & \\
= & \sigma\left(\theta C^{\prime} \vee \theta D_{1} \vee \ldots \theta D_{k}\right) & \text { by }(8) \\
= & \sigma \theta\left(C^{\prime} \vee D_{1} \vee \ldots D_{k}\right) & \\
= & \kappa \mu\left(C^{\prime} \vee D_{1} \vee \ldots D_{k}\right) & \text { by (10). }
\end{aligned}
$$

The resolution tree has the form

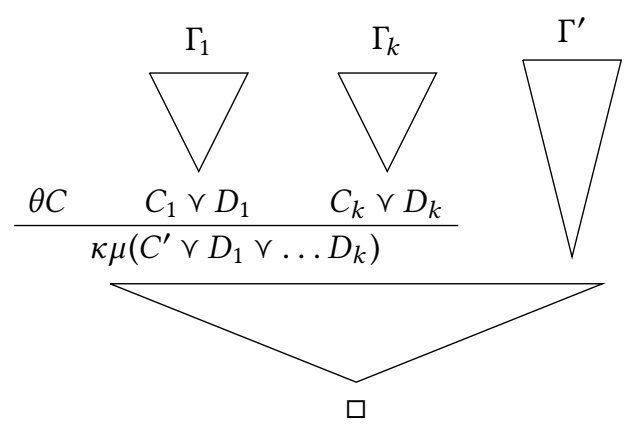

where $\Gamma=\Gamma_{1} \cup \cdots \cup \Gamma_{k} \cup \Gamma^{\prime}$.

Let us consider the resolution tree where we prune the subtree above $\kappa \mu\left(C^{\prime} \vee D_{1} \vee \ldots D_{k}\right)$. Its size is strictly smaller than $n$, its axioms are $\left\{\kappa \mu\left(C^{\prime} \vee D_{1} \vee \ldots D_{k}\right)\right\} \cup \Gamma^{\prime}$, and its conclusion is $\square$. 
Since, by definition of Resolution with Input Selection, $\mathcal{S}\left(\kappa \mu\left(C^{\prime} \vee D_{1} \vee \ldots D_{k}\right)\right)=\emptyset$, we can apply the induction hypothesis to obtain a resolution tree whose axioms are $\left\{\mu\left(C^{\prime} \vee D_{1} \vee \ldots D_{k}\right)\right\} \cup \Gamma^{\prime}$ and whose conclusion is $\square$. We can extend this tree by replacing the leaf $\mu\left(C^{\prime} \vee D_{1} \vee \ldots D_{k}\right)$ by the tree

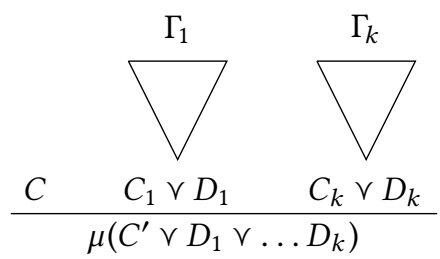

which is valid because $\mu$ is the most general solution to the unification problem $L_{1}={ }^{?} L^{\prime}$, Prob which is the one that needs to be solved for the inference rule to be applied. We therefore obtain a resolution tree whose axioms are $\{C\} \cup \Gamma_{1} \cup \cdots \cup \Gamma_{k} \cup \Gamma^{\prime}=\{C\} \cup \Gamma$ and whose conclusion is $\square$, as was expected.

LEMmA 4.6. For all sets of clauses $\Gamma$, for all substitutions $\theta$, for all clauses $C$ such that $\mathcal{S}(\theta C)=\emptyset$, if $\Gamma, \theta C \sim^{*} \square$ then $\Gamma, C \sim{ }^{*} \square$ where $\mathcal{S}(C)=\emptyset$.

Proof. Using Lemma 3.3, there exists a resolution tree whose axioms are included, as a set, in $\Gamma \cup\{\theta C\}$, and whose conclusion is $\square$. Repetitively applying Lemma 4.5, one can replace all axioms $\theta C$ by $C$, thus obtaining a resolution tree whose axioms are included, as a set, in $\Gamma \cup\{C\}$. Using Lemma 3.3 in the converse direction, we obtain $\Gamma, C \sim^{*} \square$.

THEOREM 4.7. If $\ulcorner\Gamma\urcorner \Uparrow \vdash$, then $\Gamma \sim^{*} \square$.

Proof. By induction on the proof $\ulcorner\Gamma\urcorner \Uparrow \vdash$. We generalize the statement a little by allowing the sequent to contain not only translations of clauses but also literals with a positive polarity, which are associated to the corresponding singleton clauses. On such a sequent, only two rules can be applied, namely $\widehat{\pi}_{\vdash}$ and Focus. Since $\ulcorner\Gamma\urcorner$ contains only clausal formulas and positive literals, there are only four cases:

$$
\pi_{\vdash} \frac{}{\left\ulcorner\Gamma^{\prime}\right\urcorner, L, L^{\perp} \Uparrow \vdash}
$$

In that case, we can simply apply Resolution on $L$ and $L^{\perp}$ to derive $\square$, hence $\Gamma^{\prime}, L, L^{\perp} \leadsto \square$.

- The proof focuses on a formula corresponding to the empty clause:

In that case, $\square$ already belongs to $\Gamma$.

$$
\text { Focus } \frac{\Downarrow \perp\urcorner \Downarrow \perp \vdash}{\ulcorner\Gamma\urcorner \Uparrow \vdash}
$$

- The proof focuses on a formula corresponding to a clause without selection. Because of focusing constraints, the proof is necessarily of the form:

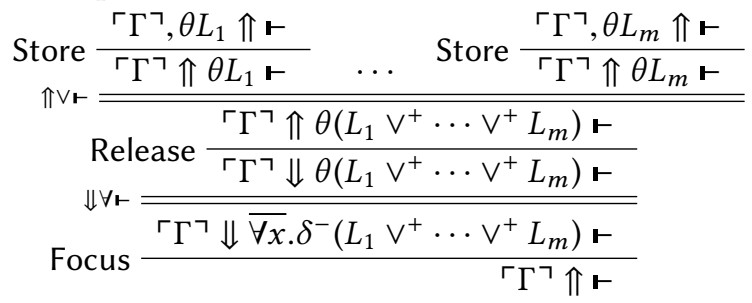

By induction hypothesis, we have derivations of $\Gamma, \theta L_{k} \sim^{*} \square$ for all $1 \leq k \leq m$. By Corollary 4.4, we have a derivation $\Gamma, \theta\left(L_{1} \vee \cdots \vee L_{k}\right) \sim^{*} \square$ with nothing selected in $\theta\left(L_{1} \vee \cdots \vee L_{k}\right)$. By Lemma 4.6, we have a derivation of $\Gamma \sim^{*} \square$, with $L_{1} \vee \cdots \vee L_{k}$ in $\Gamma$. 
- The proof focuses on a formula corresponding to a clause with selection. Because of focusing constraints, the proof is necessarily of the form:

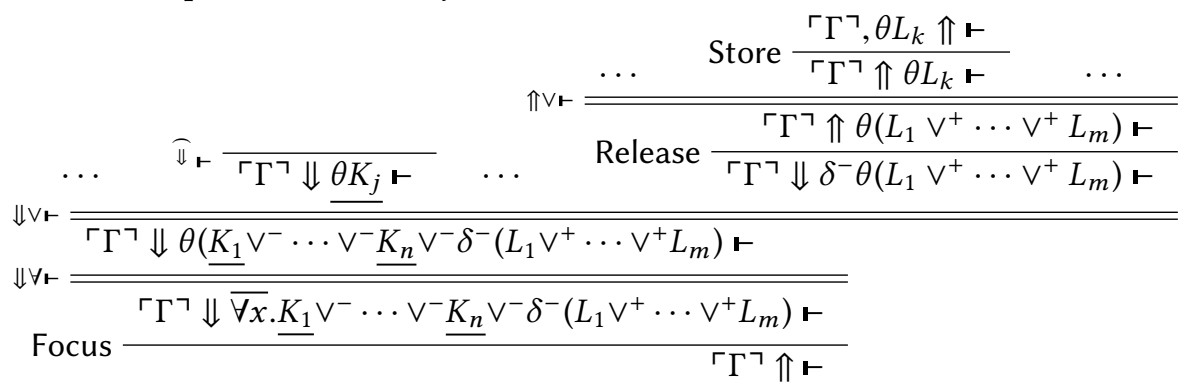

where $\overline{\forall x} \cdot \underline{K_{1}} \vee^{-} \cdots \vee^{-} K_{n} \vee^{-}\left(L_{1} \vee^{+} \ldots \vee^{+} L_{m}\right)$ and $\theta K_{j}^{\perp}$ for all $1 \leq j \leq n$ are members of $\ulcorner\Gamma\urcorner$.

By induction hypothesis, we have derivations of $\Gamma, \theta L_{k} \sim \sim^{*} \square$ for all $1 \leq k \leq m$. By Corollary 4.4, we have a derivation $\Gamma, \theta\left(L_{1} \vee \cdots \vee L_{m}\right) \sim^{*} \square$.

From $K_{1} \vee \cdots \vee \underline{K_{n}} \vee L_{1} \vee \cdots \vee L_{m}$, since the application of $\widetilde{\Downarrow}_{\mathfrak{r}}$ above imposes that all $\theta K_{j}{ }^{\perp}$

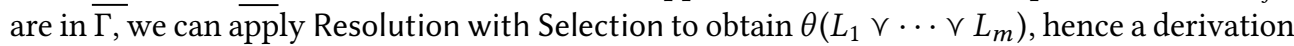
$\Gamma \leadsto * \square$.

\subsection{From Resolution Derivations to Focused Proofs}

We prove that all inference rules of Resolution with Input Selection are admissible in $\mathrm{LKF}^{\perp}$ : if $\Gamma \leadsto C$ then $\mathrm{LKF}^{\perp}$ proofs of $\ulcorner\Gamma\urcorner,\ulcorner C\urcorner \Uparrow \vdash$ can be turned into proofs of $\ulcorner\Gamma\urcorner \Uparrow \vdash$. For this direction, we do not need to assume that generated clauses do not select any literal: if a derivation is found in Resolution with selection, it would be translated into $\mathrm{LKF}^{\perp}$ even if some literals are selected in generated clauses, as long as selection is compatible with instantiation, i.e. $\sigma \mathcal{S}(C) \subseteq \mathcal{S}(\sigma C)$ for all clauses $C$ and substitutions $\sigma$. (Note that we do not need the converse inclusion.)

Lemma 4.8. For all sets of formulas $\Gamma$, for all clauses $C$, for all substitutions $\sigma$, assuming $\sigma \mathcal{S}(C) \subseteq$ $\mathcal{S}(\sigma C)$, if $\Gamma,\ulcorner C\urcorner,\ulcorner\sigma C\urcorner \Uparrow \vdash$, then $\Gamma,\ulcorner C\urcorner \Uparrow \vdash$.

Proof. By induction on the proof $\Gamma,\ulcorner C\urcorner,\ulcorner\sigma C\urcorner \Uparrow \vdash$. Note that to be able to apply the induction hypothesis directly, one needs to have a sequent of the form $\Gamma^{\prime} \Uparrow \vdash$, so that one considers in general coarse grain proof steps consisting of an alternation of a synchronous and an asynchronous phases.

If the proof does not begin by acting on $\ulcorner\sigma C\urcorner$, this is a simple application of the induction hypothesis. Otherwise, note that the only rule that can act on $\ulcorner\sigma C\urcorner$ is Focus: even in the case when $\sigma C$ consists only of a single literal, $\ulcorner\sigma C\urcorner$ is $\delta^{-}(\sigma C)$ so that $\widehat{\pi}_{\vdash}$ cannot be applied. Let $\sigma C$ be $\underline{K_{1}} \vee \cdots \vee \underline{K_{n}} \vee L_{1} \vee \cdots \vee L_{m}$. The proof begins with

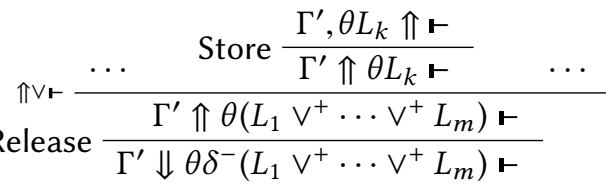

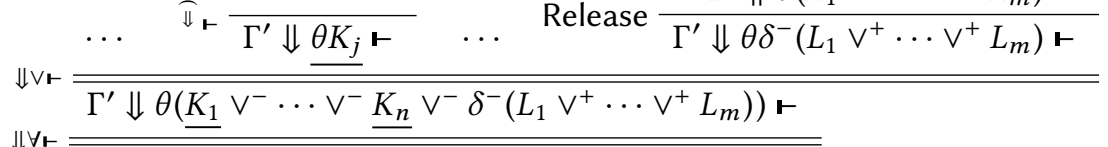

$$
\begin{aligned}
& \text { Focus } \frac{\overline{\Gamma^{\prime} \Downarrow \overline{\forall x} . \underline{K_{1}} \vee^{-} \cdots \vee^{-} \underline{K_{n}} \vee^{-} \delta^{-}\left(L_{1} \vee^{+} \cdots \vee^{+} L_{m}\right) \vdash}}{\Gamma^{\prime} \Uparrow \vdash}
\end{aligned}
$$

where $\Gamma^{\prime}=\Gamma,\ulcorner C\urcorner,\ulcorner\sigma C\urcorner$ and for all $1 \leq j \leq n$ the literal $\theta K_{j}{ }^{\perp}$ is in $\Gamma$. 
Let $C$ be $K_{1}^{1} \vee \cdots K_{1}^{k_{1}} \vee \cdots K_{n}^{1} \vee \cdots K_{n}^{k_{n}} \vee L_{1}^{1} \vee \cdots L_{1}^{l_{1}} \vee \cdots L_{m}^{1} \vee \cdots L_{m}^{l_{m}}$ where for all $i, j$ we have $\sigma K_{i}^{j}=K_{i}$ and $\sigma L_{i}^{j}=L_{i}$. By hypothesis, the literals selected in $C$ are among the $K_{i}^{j}$ (hence the dashed underline). For all $i, j$, one can either build a proof $\rrbracket_{\vdash} \overline{\Gamma,\ulcorner C\urcorner \Downarrow \theta \sigma K_{i}^{j} \vdash}$ if it is selected, or Store $\frac{\pi_{\vdash,\ulcorner C\urcorner, \theta \sigma K_{i}^{j} \Uparrow \vdash}}{\Gamma,\ulcorner C\urcorner \Uparrow \theta \sigma K_{i}^{j} \vdash}$ if it is not.

We apply the induction hypothesis on $\Gamma^{\prime}, \theta L_{k} \Uparrow \vdash$, hence we have proofs $\Gamma,\ulcorner C\urcorner, \theta L_{k} \Uparrow \vdash$ for all $k$. We can therefore build the proof

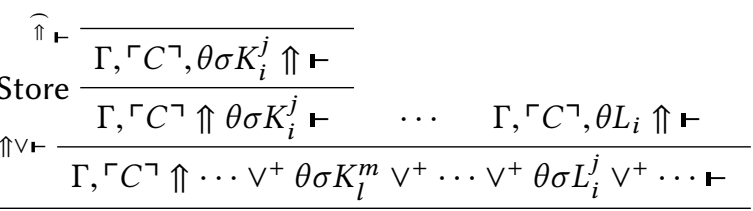

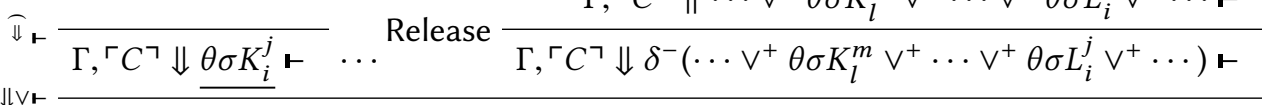

$$
\begin{aligned}
& \Gamma,\ulcorner C\urcorner \Downarrow \cdots \vee^{-} \theta \sigma K_{i}^{j} \vee^{-} \cdots \vee^{-} \delta^{-}\left(\cdots \vee^{+} \theta \sigma K_{l}^{m} \vee^{+} \cdots \vee^{+} \theta \sigma L_{i}^{j} \vee^{+} \cdots\right) \vdash \\
& \Downarrow \forall \vdash \overline{\Gamma,\ulcorner C\urcorner \Downarrow \overline{\forall x} . \cdots \vee^{-} \underline{K_{i}^{j}} \vee^{-} \cdots \vee^{-} \delta^{-}\left(\cdots \vee^{+} K_{l}^{m} \vee^{+} \cdots \vee^{+} L_{i}^{j} \vee^{+} \cdots\right) \vdash} \\
& \text { Focus } \\
& \Gamma,\ulcorner C\urcorner \Uparrow \vdash
\end{aligned}
$$

Corollary 4.9. Factoring is admissible in $L K F^{\perp}$.

LEMMA 4.10. Resolution is admissible in $L K F^{\perp}$ :

For all sets of formulas $\Gamma$, for all clauses $L \vee C$ and $L^{\prime \perp} \vee D$ without selection, if $\sigma=\operatorname{mgu}\left(L, L^{\prime}\right)$, if $\Gamma,\ulcorner L \vee C\urcorner,\left\ulcorner L^{\prime \perp} \vee D\right\urcorner,\ulcorner\sigma(C \vee D)\urcorner \Uparrow \vdash$ then $\Gamma,\ulcorner L \vee C\urcorner,\left\ulcorner L^{\prime \perp} \vee D\right\urcorner \Uparrow \vdash$.

Proof. By induction on the proof $\Gamma,\ulcorner L \vee C\urcorner,\left\ulcorner L^{\prime \perp} \vee D\right\urcorner,\ulcorner\sigma(C \vee D)\urcorner \Uparrow \vdash$.

Let $\Gamma^{\prime}$ be $\Gamma,\ulcorner L \vee C\urcorner,\left\ulcorner L^{\prime \perp} \vee D\right\urcorner$ and $\Gamma^{\prime \prime}$ be $\Gamma^{\prime},\ulcorner\sigma(C \vee D)\urcorner$. If the proof does not begin by focusing on $\ulcorner\sigma(C \vee D)\urcorner$, this is a simple application of the induction hypothesis. Otherwise, let $\sigma(C \vee D)$ be $\underline{I_{1}} \vee \cdots \vee \underline{I_{n}} \vee J_{1} \vee \cdots \vee J_{m}$. The proof begins with

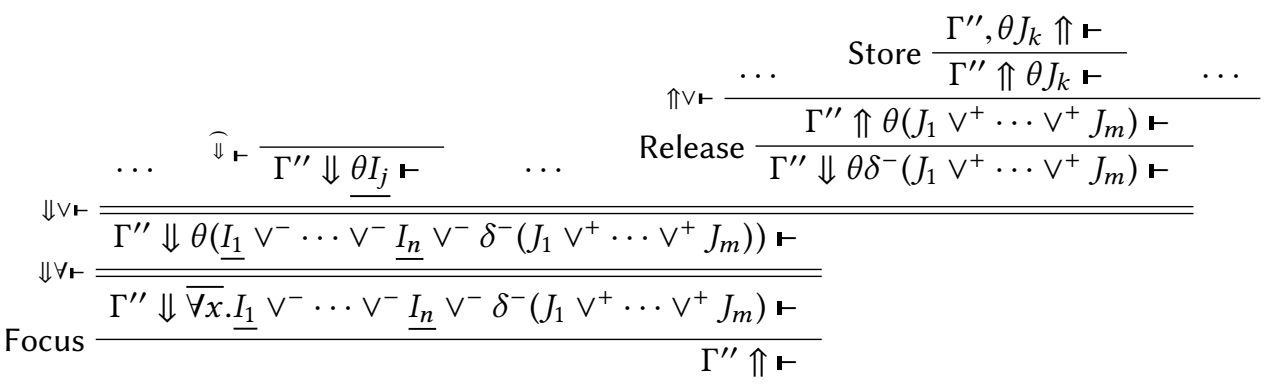

where, to be able to close the left branches, for all $1 \leq j \leq n$ the literal $\theta I_{j}{ }^{\perp}$ is in $\Gamma^{\prime}$.

We know that $C$ is $\cdots \vee I_{i}^{1} \vee \cdots I_{i}^{k_{i}} \vee \cdots \vee J_{j}^{1} \vee \cdots J_{j}^{l_{j}} \vee \cdots$ where $i$ ranges over a subset of $\{1, \ldots, n\}$ and $j$ over a subset of $\{1, \ldots, m\}$, and $\sigma I_{i}^{x}=I_{i}$ and $\sigma J_{j}^{y}=J_{j}$ for all $x, y$. Likewise, $D$ is $\cdots \vee I_{i}^{\prime 1} \vee \cdots I_{i}^{\prime k_{i}} \vee \cdots \vee J_{j}^{\prime 1} \vee \cdots J_{j}^{\prime l_{j}} \vee \cdots$ where $i$ ranges over a subset of $\{1, \ldots, n\}$ and $j$ over a subset of $\{1, \ldots, m\}$, and $\sigma I_{i}^{\prime x}=I_{i}$ and $\sigma J_{j}^{\prime y}=J_{j}$ for all $x, y$.

We apply the induction hypothesis on $\Gamma^{\prime \prime}, \theta J_{k} \Uparrow \vdash$, hence we have a proof of $\Gamma,\ulcorner L \vee C\urcorner,\left\ulcorner L^{\prime \perp} \vee D\right\urcorner, \theta J_{k} \Uparrow$. 
We can build the following proof of $\Gamma^{\prime} \Uparrow \vdash$ : first, we focus on $\ulcorner L \vee C\urcorner$ to get the derivation

$$
\begin{aligned}
& \text { Store } \frac{\Gamma^{\prime}, \theta \sigma L \Uparrow \vdash}{\Gamma^{\prime} \Uparrow \theta \sigma L \vdash} \quad \ldots \quad \text { Store } \frac{\Gamma^{\prime}, \theta I_{i} \Uparrow \vdash}{\Gamma^{\prime} \Uparrow \theta I_{i} \vdash} \quad \ldots \quad \text { Store } \frac{\Gamma^{\prime}, \theta J_{j} \Uparrow \vdash}{\Gamma^{\prime} \Uparrow \theta J_{j} \vdash} \ldots \\
& \text { Release } \frac{\Gamma^{\prime} \Uparrow \theta \sigma\left(L \vee^{+} \ldots \vee^{+} I_{i}^{1} \vee^{+} \ldots I_{i}^{k_{i}} \vee^{+} \ldots \vee^{+} J_{j}^{1} \vee^{+} \ldots J_{j}^{l_{j}} \vee^{+} \ldots\right) \vdash}{\Gamma^{\prime} \Downarrow \theta \sigma \delta^{-}\left(L \vee^{+} \ldots \vee^{+} I_{i}^{1} \vee^{+} \ldots I_{i}^{k_{i}} \vee^{+} \ldots \vee^{+} J_{j}^{1} \vee^{+} \ldots J_{j}^{l_{j}} \vee^{+} \ldots\right) \vdash} \\
& \text { Focus } \frac{\Gamma^{\prime} \Downarrow \overline{\forall x} . \delta^{-}\left(L \vee^{+} \ldots \vee^{+} I_{i}^{1} \vee^{+} \cdots I_{i}^{k_{i}} \vee^{+} \ldots \vee^{+} J_{j}^{1} \vee^{+} \ldots J_{j}^{l_{j}} \vee^{+} \ldots\right) \vdash}{\Gamma^{\prime} \Uparrow \vdash}
\end{aligned}
$$

In this derivation, all right branches are closed by induction hypothesis. On the left branch, we focus on $\left\ulcorner L^{\prime \perp} \vee D\right\urcorner$. Let $\Gamma^{\prime \prime \prime}$ be $\Gamma^{\prime}, \theta \sigma L$. We get the derivation

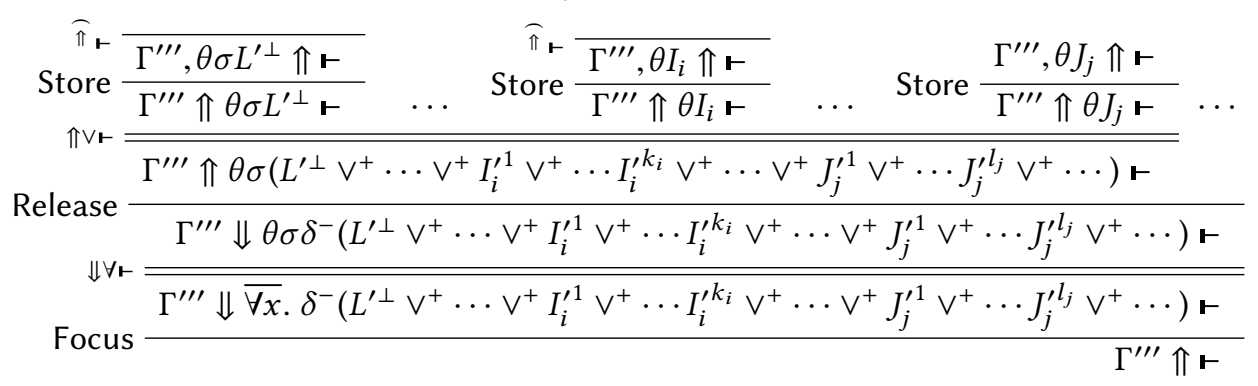

The left branch can be closed because $\theta \sigma L=\theta \sigma L^{\prime}$. The right branches are closed by induction hypothesis.

LemMa 4.11. Resolution with Selection is admissible in $L K F^{\perp}$ :

For all sets of formulas $\Gamma$, for all clauses $K_{1} \vee \ldots \vee K_{n} \vee C, K_{1}^{\prime \perp} \vee D_{1}, \ldots$, and $K_{n}^{\prime \perp} \vee D_{n}$, where $\mathcal{S}\left(\underline{K_{1}} \vee \ldots \vee \underline{K_{n}} \vee C\right)=\left\{K_{1} ; \ldots ; K_{n}\right\}, \mathcal{S}\left(\overline{K_{i}^{\prime \perp}} \vee D_{i}\right) \overline{=\emptyset}$ and $\sigma$ is the most general unifier of the simultaneous unification problem $K_{1}=?$ ? $K_{1}^{\prime}, \ldots, K_{n}=?$ ? $K_{n}^{\prime}$ if $\Gamma,\left\ulcorner K_{1} \vee \ldots \vee K_{n} \vee C\right\urcorner, \ldots\left\ulcorner K_{i}^{\prime \perp} \vee D_{i}\right\urcorner, \ldots,\left\ulcorner\sigma\left(C \vee D_{1} \vee \cdots \vee D_{n}\right)\right\urcorner \Uparrow \vdash$ then $\left.\overline{\Gamma,\ulcorner} K_{1} \vee \ldots \vee \underline{K_{n}} \vee C\right\urcorner, \ldots\left\ulcorner K_{i}^{\prime \perp} \vee D_{i}\right\urcorner, \ldots \Uparrow \vdash$.

Proof. By induction on the proof

$\Gamma,\left\ulcorner K_{1} \vee \ldots \vee K_{n} \vee C\right\urcorner, \ldots,\left\ulcorner K_{i}^{\perp} \vee D_{i}\right\urcorner, \ldots,\left\ulcorner\sigma\left(C \vee D_{1} \vee \ldots \vee D_{n}\right)\right\urcorner \Uparrow \vdash$. We follow the same idea as in the proofs of the two precedent lemmas.

If the proof does not begin by focusing on $\left\ulcorner\sigma\left(C \vee D_{1} \vee \cdots \vee D_{n}\right)\right\urcorner$, this is a simple application of the induction hypothesis. Otherwise, let $\Gamma^{\prime}$ be $\Gamma,\left\ulcorner K_{1} \vee \ldots \vee \underline{K_{n}} \vee C\right\urcorner, \ldots\left\ulcorner K_{i}^{\prime \perp} \vee D_{i}\right\urcorner, \ldots$ and $\Gamma^{\prime \prime}$ be $\Gamma^{\prime},\left\ulcorner\sigma\left(C \vee D_{1} \vee \cdots \vee D_{n}\right)\right\urcorner$. Focusing leads us either to sequents $\Gamma^{\prime \prime} \Downarrow \theta I_{j} \vdash$, with $\theta I_{j}{ }^{\perp}$ in $\Gamma$, or to sequents $\Gamma^{\prime \prime}, \theta J_{k} \Uparrow \vdash$ upon which one can apply the induction hypothesis. Let us remark that for each literal $L$ of $C$ or $D_{i}, \theta \sigma L$ is either one of $\theta I_{j}$ or one of $\theta J_{k}$. Therefore, we know how to close proofs of $\Gamma^{\prime} \Uparrow \theta \sigma L \vdash$ for each, either by induction hypothesis or using $\theta I_{j}{ }^{\perp}$ in $\Gamma$.

To build the proof of $\Gamma^{\prime} \Uparrow \vdash$, we first focus on $\left\ulcorner K_{1}^{\prime \perp} \vee D_{1}\right\urcorner$, instantiating the variables using the substitution $\theta \sigma$. As explained above, we know how to close the branches coming from $D_{1}$, it remains the branch $\Gamma^{\prime}, \theta \sigma K_{1}^{\prime \perp} \Uparrow \boldsymbol{r}$.

We do the same, focusing on $\left\ulcorner K_{2}^{\prime \perp} \vee D_{2}\right\urcorner$ then ... then $\left\ulcorner K_{n}^{\prime \perp} \vee D_{n}\right\urcorner$ and the remaining branch is $\Gamma^{\prime}, \theta \sigma K_{1}^{\prime \perp}, \ldots \theta \sigma K_{n}^{\prime \perp} \Uparrow \vdash$.

We can close the proof by focusing on $\left\ulcorner K_{1} \vee \ldots \vee K_{n} \vee C\right\urcorner$. Branches coming from $C$ can be closed as before, and the other branches are closed by 


$$
\bar{\Downarrow}_{\vdash} \overline{\Gamma^{\prime}, \theta \sigma K_{1}^{\prime \perp}, \ldots \theta \sigma K_{n}^{\prime \perp} \Downarrow \underline{\theta \sigma K_{i}} \vdash}
$$

since $\theta \sigma K_{i}^{\prime}=\theta \sigma K_{i}$ for all $i$.

THEOREM 4.12. For all sets of clauses $\Gamma$, if $\Gamma \sim^{*} \square$, then $\ulcorner\Gamma\urcorner \Uparrow$.

Proof. By induction on the length of the derivation $\Gamma \sim^{*} \square$. If $\square$ is in $\Gamma$, then we focus on $\ulcorner\square\urcorner=\perp$ and apply $\Downarrow \perp r$. If the first step is Factoring, we apply Lemma 4.8. If it is Resolution, we apply Lemma 4.10. If it is Resolution with Selection, we apply Lemma 4.11.

Note that the rules of resolution with input selection are admissible, but they are not derivable. In particular, the size of the proof in $\mathrm{LKF}^{\perp}$ can be much larger than the resolution derivation, as expected in a cut-free sequent calculus. Using cuts would lead to a closer correspondence between resolution derivations and sequent-calculus proofs, as in [18]. However, we chose to stay in the cut-free fragment to prove that, even in the incomplete case, resolution coincides with cut-free proofs, as in [29].

\section{COMPLETE INSTANCES}

\subsection{Ordinary Focusing and Semantic Hyperresolution}

As said earlier, in standard LKF, not all occurrences of literals can have an arbitrary polarity. Instead, each atomic formula $P$ is given globally a polarity, and $P^{\perp}$ has the opposite polarity.

Let us first look at the simple case where atomic formulas are given a positive polarity. We recall the completeness theorem of LKF:

Theorem 5.1 (CoRollary of [31, Theorem 17]). If the literals with a positive polarity are exactly the atomic formulas, $L K F^{\perp}$ is (sound and) complete.

If we look at the corresponding resolution calculus, Resolution with Selection for this particular instance becomes:

$$
\text { R.w.S. } \frac{\neg P_{1} \vee \ldots \vee \neg P_{n} \vee C \quad P_{1}^{\prime} \vee D_{1} \quad \ldots \quad P_{n}^{\prime} \vee D_{n}}{\sigma\left(C \vee D_{1} \vee \ldots \vee D_{n}\right)}
$$

where $C$ and $D_{i}$ for all $i$ contain only positive literals, and $\sigma$ is the most general unifier of $P_{1}=$ ? $P_{1}^{\prime}, \ldots, P_{n}=? P_{n}^{\prime}$. Note that the clause $\sigma\left(C \vee D_{1} \vee \ldots \vee D_{n}\right)$ contains only atomic formulas, and in agreement with the definition of Resolution with Selection, they have a positive polarity; therefore, no literal would be selected in it even if it was an input clause. Besides, Resolution cannot be applied, since there exists no clause $\neg P \vee C$ with $\mathcal{S}(\neg P \vee C)=\emptyset$.

This corresponding resolution calculus is therefore exactly hyperresolution of Robinson [34]: premises of an inference contain only positive literals, except one clause whose all negative literals are resolved at once. Theorem 4.2 therefore links ordinary focusing with hyperresolution. Consequently, Theorem 5.1 implies the completeness of hyperresolution.

Chaudhuri et al. [16, Theorem 16] prove a similar result by establishing a correspondence between hyperresolution derivations and proofs in a focused sequent calculus for intuitionistic linear logic, but only considering Horn clauses. In their setting, choosing a negative polarity for atomic formulas leads to SLD resolution, which is the reasoning mechanism of Prolog. Note that in our case, choosing a negative polarity for atomic formulas leads to what is called negative hyperresolution, which is the same as hyperresolution but with the role of positive and negative literals inversed. The difference with [16] is that it is concerned with intuitionistic logic, so that positive and negative literals are not dual. 
Let us now look at the general case, where atomic formulas are given an arbitrary polarity. Let us first stick to the ground case. We recall a refinement of resolution called Semantic hyperresolution [36][14, Sect. 1.3.5.3]. Let $I$ be an arbitrary Herbrand interpretation, i.e. a model whose domain is the set of terms interpreted as themselves. Note that $I$ is not assumed to be a model of the input set of clauses (which is fortunate, since one is trying to show that it is unsatisfiable). Given a clause $C$, the idea of semantic hyperresolution is to resolve all literals of $C$ that are valid in $I$ at once, with clauses in which all literals are not valid in $I$. This gives the rule:

$$
\operatorname{SHR} \frac{K_{1} \vee \ldots \vee K_{n} \vee C \quad K_{1}{ }^{\perp} \vee D_{1} \quad \ldots \quad K_{n}{ }^{\perp} \vee D_{n}}{C \vee D_{1} \vee \ldots \vee D_{n}}
$$

where for all $i, I \mid=K_{i}$ (and thus $I \not \notin K_{i}^{\perp}$ ), $I \not \notin C$ and $I \not \models D_{i}$. Note that $I \not \models C \vee D_{1} \vee \ldots \vee D_{n}$.

Semantic hyperresolution for a Herbrand interpretation $I$ can be seen as an instance of Resolution with Input Selection by using the following polarization of atomic formulas: a literal $L$ has a negative polarity iff $I=L$. In that case, SHR corresponds exactly to Resolution with Selection, and Resolution cannot be applied since we cannot have clauses $P \vee C$ and $\neg P \vee D$ where both $P$ and $\neg P$ are not valid in $I$.

This particular instance of polarization is in fact the ordinary version of focusing. Indeed, once a global polarity is assigned to each atomic formula, the set of literals whose polarity is negative defines a Herbrand interpretation, and we saw reciprocally how to design a global polarization from the Herbrand interpretation. Theorem 4.2 therefore links ordinary focusing in the ground case with semantic hyperresolution. They are both complete, thanks to this theorem:

Theorem 5.2 (Corollary of [31, Theorem 17]). Given a global polarization of atomic formulas, where the polarity of $P^{\perp}$ is the opposite of that of $P, L K F^{\perp}$ is (sound and) complete.

Completeness of LKF therefore leads to proofs of completeness of semantic hyperresolution in the ground case, and hyperresolution in the first-order case, that do not rely on the construction of a model. To our knowledge, in addition to Chaudhuri et al. [16], only Goubault-Larrecq [27] showed a similar result. We also provided a proof similar to the one in this paper, but restricted to Ordered Resolution, without selection of literals [6].

Note that we cannot extend this link between semantic hyperresolution and focusing to the non-ground case. Indeed, in the non-ground case, $I=L$ iff $I$ is a model for all ground instances of $L$. Hence, given a literal $L$ such that $I \not k L$, we can nonetheless have an instance $\sigma L$ such that $I=\sigma L$; $\sigma L$ should therefore be selected in the generated clause, but our resolution calculus cannot take this into account. However, remark that if $I \mid=L$ then $I \mid=\sigma L$, so that $\sigma(\mathcal{S}(C)) \subseteq \mathcal{S}(\sigma C)$. Thus, the condition of Lemma 4.8 is fulfilled. Therefore, even in the non-ground case, Theorem 4.12 can be used to show that the completeness of semantic hyperresolution implies the completeness of LKF.

\subsection{Deduction Modulo Theory}

Deduction Modulo Theory [23] is a framework that consists in applying the inference rules of an existing proof system modulo some congruence over formulas. This congruence represents the theory, and it is in general defined by means of rewriting rules. To be expressive enough, these rules are defined not only at the term level, but also for formulas. To get simpler presentations of theories, we distinguish between rewrite rules that can be applied at positive and at negative positions by giving them a polarity ${ }^{3}$, where by negative position we mean under an odd number of $\neg$. We therefore have positive rules $P \rightarrow^{+} A$ and negative rules $P \rightarrow^{-} A$ where $P$ is an atomic formula and $A$ an arbitrary formula whose free variables appear in $P$. The rewrite relation $B_{1} \stackrel{+}{\longrightarrow} B_{2}$ is defined by saying that there exists either a positive position $\mathfrak{p}$ and a positive rule $P \rightarrow^{+} A$, or

\footnotetext{
${ }^{3}$ This polarity must not be confused with the other notions of polarity mentioned in the paper.
} 


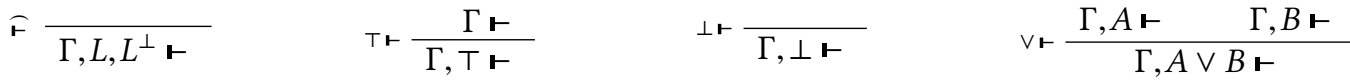

$$
\begin{aligned}
& \wedge \vdash \frac{\Gamma, A, B \vdash}{\Gamma, A \wedge B \vdash} \quad \exists \vdash \frac{\Gamma, A \vdash}{\Gamma, \exists x \cdot A \vdash} x \text { not free in } \Gamma \quad \forall \vdash \frac{\Gamma, \forall x \cdot A,\{t / x\} A \vdash}{\Gamma, \forall x \cdot A \vdash} \\
& \uparrow^{-} \vdash \frac{\Gamma, P, A \vdash}{\Gamma, P \vdash} P \stackrel{-}{\longrightarrow} A \quad \uparrow^{+} \vdash \frac{\Gamma, \neg P, A^{\perp} \vdash}{\Gamma, \neg P \vdash} P \stackrel{+}{\longrightarrow} A
\end{aligned}
$$

Fig. 3. The sequent calculus PUSC ${ }^{\perp}$

a negative position $\mathfrak{p}$ and a negative rule $P \rightarrow^{-} A$; and a substitution $\sigma$ such that the subformula of $B_{1}$ at position $\mathfrak{p}$ is $\sigma P$ and $B_{2}$ equals $B_{1}$ where the subformula at position $\mathfrak{p}$ is replaced by $\sigma A$. $\longrightarrow$ is defined dually (negative position and positive rule, or positive position and negative rule). In Polarized Sequent Calculus Modulo theory [20], the inference rules of the sequent calculus are applied modulo such a polarized rewriting system, as for instance in

$$
\vdash \frac{\Gamma \vdash A, \Delta \quad \Gamma \vdash B, \Delta}{\Gamma \vdash C, \Delta} C \stackrel{+}{\rightarrow} * A \wedge B .
$$

Note that the implicit semantics of a negative rule $P \rightarrow^{-} A$ is therefore $\overline{\forall x}$. $(P \Rightarrow A)$, whereas the semantics of $P \rightarrow^{+} A$ is $\overline{\forall x}$. $(A \Rightarrow P)$, where $\bar{x}$ are the free variables of $P$.

With Kirchner [12], we proved the equivalence of Polarized Sequent Calculus Modulo theory to a sequent calculus where polarized rewriting rules are applied only on literals, using explicit rules. This calculus, Polarized Unfolding Sequent Calculus, is almost the calculus PUSC ${ }^{\perp}$ presented in Figure 3. The only difference is that all formulas are put on the left of the sequent in $\mathrm{PUSC}^{\perp}$. We denote by $\Gamma \vdash \mathcal{R}$ the fact that $\Gamma \vdash$ can be proved in $\mathrm{PUSC}^{\perp}$ using the polarized rewriting system $\mathcal{R}$. Note that the rule for the universal quantifier $\forall r$ as well as the unfolding rules $\uparrow^{-} r$ and $\uparrow^{+} r$ contain an implicit contraction rule, as in the sequent calculus G4 of Kleene, in order to ensure that all rules of $\mathrm{PUSC}^{\perp}$ are invertible.

We can translate polarized rewriting rules as formulas with selection, and see $\mathrm{PUSC}^{\perp}$ as an instance of $\mathrm{LKF}^{\perp}$. We first consider how to translate formulas of the right-hand side of polarized rewriting rules. We polarize them by choosing positive connectives for $\vee$ and $\wedge$ and, to unchain the introduction of the universal quantifier, we introduce delays. (Let us recall that a delay $\delta^{+}$allows to force a formula to be positive, and it can be encoded using an existential quantifier.) This gives the translation:

$$
\begin{aligned}
& |L|=L \quad \text { when } L \text { is } \top, \perp \text { or a literal } \\
& |A \vee B|=|A| \vee^{+}|B| \quad|\exists x . A|=\exists x .|A| \\
& |A \wedge B|=|A| \wedge^{+}|B| \\
& |\forall x . A|=\forall x . \delta^{+}|A|
\end{aligned}
$$

Definition 5.3. Given a negative rewriting rule $P \rightarrow^{-} A$ where the free variables of $P$ are $x_{1}, \ldots, x_{n}$, its translation as a formula with selection is $\| P \rightarrow^{-} A \rrbracket=\forall x_{1} \ldots x_{n} \cdot \neg P \vee^{-} \delta^{+}|A|$.

Given a positive rewriting rule $P \rightarrow^{+} A$ where the free variables of $P$ are $x_{1}, \ldots, x_{n}$, its translation as a formula with selection is $\| P \rightarrow^{+} A \rrbracket=\forall x_{1} \ldots \forall x_{n} \cdot \underline{P} \vee^{-} \delta^{+}\left|A^{\perp}\right|$.

The translation $\llbracket \mathcal{R} \rrbracket$ of a polarized rewriting system $\mathcal{R}$ is the multiset of the translation of its rules.

Definition 5.4. Let $A$ be a non-polarized formula. $A$ is called contractible if its top connective is $\forall$ or $\perp$, or if it is a literal.

Definition 5.5. Let $N_{1}, \ldots, N_{n}$ be a multiset of contractible formulas, and let $P_{1}, \ldots, P_{m}$ be a multiset of non-contractible formulas, then the translation of the PUSC ${ }^{\perp}$ sequent $N_{1}, \ldots, N_{n}, P_{1}, \ldots, P_{m} \vdash$ modulo the rewriting system $\mathcal{R}$ is the $\mathrm{LKF}^{\perp}$ sequent $\llbracket \mathcal{R} \rrbracket,\left|N_{1}\right|, \ldots,\left|N_{n}\right| \Uparrow\left|P_{1}\right|, \ldots,\left|P_{m}\right| r$. 
Theorem 5.6. $N_{1}, \ldots, N_{n}, P_{1}, \ldots, P_{m} \vdash \mathcal{R}$ in PUSC $C^{\perp}$ iff $\llbracket \mathcal{R} \rrbracket,\left|N_{1}\right|, \ldots,\left|N_{n}\right| \Uparrow\left|P_{1}\right|, \ldots,\left|P_{m}\right| \vdash$ in $L K F^{\perp}$.

Proof. First, one proves that, in PUSC ${ }^{\perp}$, the rules $\perp r, \forall \vdash$ and $\uparrow_{r}$ can be delayed until the other rules are no longer applicable. This can be done by showing that these rules permute with the other ones, as done by Hermant [29]. Note that this fact can be related with the strategy used in Tamed [4], a tableaux method based on Deduction Modulo Theory, where rules for universal quantifiers and for rewriting are applied when no other rules can be.

With this proviso, proofs in both calculi correspond almost exactly. $\top \vdash, \wedge$,,$\vee \vdash$ and $\exists r$ in $\mathrm{PUSC}^{\perp}$

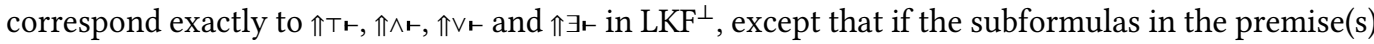
are contractible, they have to be put on the left hand side of $\Uparrow$ using Store.

The translation of $\perp_{r}$ corresponds to $\Downarrow \perp_{r}$, except that the latter can only be applied when there are only contractible formulas, which is the case if $\perp \vdash$ has been delayed as mentioned above:

$\perp \vdash \frac{}{N_{1}, \ldots, \perp, \ldots, N_{n} \vdash}$ becomes Focus $\frac{\llbracket \mathcal{R} \rrbracket,\left|N_{1}\right|, \ldots, \perp, \ldots,\left|N_{n}\right| \Downarrow \perp \vdash}{\llbracket \mathcal{R} \rrbracket,\left|N_{1}\right|, \ldots, \perp, \ldots,\left|N_{n}\right| \Uparrow \vdash}$. Conversely, if a proof in $\mathrm{LKF}^{\perp}$ starts by focusing on $\perp$, then necessarily it is immediately closed by $\Downarrow \perp r$, which is translated to $\perp \leftarrow$ in PUSC $^{\perp}$.

Similarly, $\forall \mathfrak{r}$ corresponds to $\Downarrow \forall_{\mathfrak{r}}$, with the same condition that there are only contractible formulas: $\forall \vdash \frac{N_{1}, \ldots, N_{n}, \forall x . A,\{t / x\} A \vdash}{N_{1}, \ldots, N_{n}, \forall x . A \vdash}$ becomes

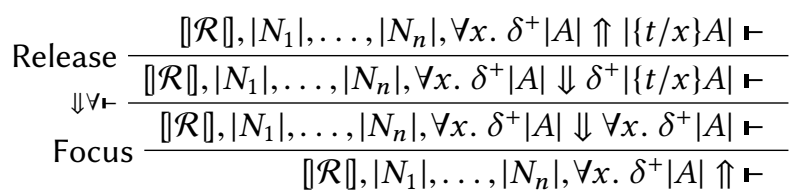

with an extra Store step if $\{t / x\} A$ is contractible. Here again, conversely, if the proof in $\mathrm{LKF}^{\perp}$ starts by focusing on the translation of a formula whose top connective is $\forall$, then it is necessarily of the shape above, which corresponds to $\forall$ r in $\mathrm{PUSC}^{\perp}$.

For the unfolding rules, if $P$ rewrites positively to $A$, then there exists a rule $Q \rightarrow^{+} B$ and a substitution $\theta$ such that $P=\theta Q$ and $A=\theta B$. This rule corresponds to a formula $\square Q \rightarrow^{+}$ $B[]=\overline{\forall x} . Q \vee^{-} \delta^{+}\left|B^{\perp}\right|$. Always with the proviso that there are only contractible formulas, let $\Gamma=\llbracket \mathcal{R}^{\prime} \rrbracket, \overline{\overline{\forall x}} . \underline{Q} \vee^{-} \delta^{+}\left|B^{\perp}\right|,\left|N_{1}\right|, \ldots,\left|N_{n}\right|, \neg P$, then $\uparrow^{+} \vdash$ therefore corresponds to

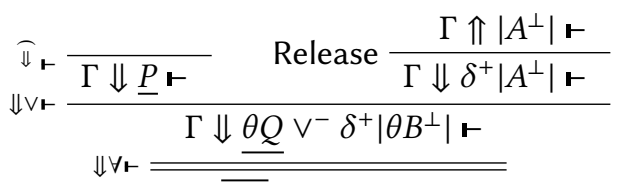

$$
\begin{aligned}
& \text { Focus } \frac{\Gamma \Downarrow \overline{\forall x} \cdot \underline{Q} \vee^{-} \delta^{+}\left|B^{\perp}\right| \vdash}{\Gamma \Uparrow \vdash}
\end{aligned}
$$

with an extra Store step if $\left|A^{\perp}\right|$ is contractible. Conversely, let us look how the encoding of a rewriting rule $Q \rightarrow^{+} B$, namely $\overline{\forall x} \cdot \underline{Q} \vee^{-} \delta^{+}\left|B^{\perp}\right|$, can be involved in a $\mathrm{LKF}^{\perp}$ proof. Given a sequent $\llbracket \mathcal{R}^{\prime} \rrbracket, \overline{\forall x} . Q \vee^{-} \delta^{+}\left|B^{\perp}\right|, \Gamma \Uparrow \vdash$, the only way to use $\overline{\forall x .} Q \vee^{-} \delta^{+}\left|B^{\perp}\right|$ is to apply a Focus on it. In that case, the derivation is necessarily of the same shape as above, so, in order to close it, we know that there must be a literal $\neg \theta Q$ in $\Gamma$. Therefore, the derivation corresponds to an unfolding of $\neg \theta Q$ into $\left|\theta B^{\perp}\right|$ in $\mathrm{PUSC}^{\perp}$.

The case of a negative rewriting is dual. 


$$
\begin{array}{cc}
\text { Resolution } \frac{P \vee C \quad \neg Q \vee D}{\sigma(C \vee D)}{ }^{a} & \text { Factoring } \frac{L \vee K \vee C}{\sigma(L \vee C)} \sigma=m g u(L, K) \\
\text { Ext. Narr. }{ }^{-} \frac{P \vee C}{\sigma(D \vee C)}{ }^{a}, Q \rightarrow^{-} D & \text { Ext. Narr. }{ }^{+} \frac{\neg Q \vee D}{\sigma(C \vee D)}{ }^{a}, P \rightarrow^{+} \neg C \\
{ }^{a} \sigma=m g u(P, Q) &
\end{array}
$$

Fig. 4. Inference rules of Polarized Resolution Modulo theory

Let us now consider the subcase where the rewriting rules are clausal, according to the terminology of Dowek [22], e.g. they are of the form $P \rightarrow^{-} C$ or $P \rightarrow^{+} \neg C$ for some clausal formula $C$. In that case, the resolution method based on Deduction Modulo Theory [23] can be refined into what is called Polarized Resolution Modulo theory [22], whose rules are given in Fig. 4. (A refinement of) Polarized Resolution Modulo theory is actually implemented in the automated theorem prover iProverModulo [7].

By noting that the translation of the rule $Q \rightarrow^{-} D$ is $\llbracket Q \rightarrow^{-} D \rrbracket=\forall x_{1} \ldots \forall \forall x_{n} . \neg Q \vee^{-} \delta^{+}|D|$ whereas $\ulcorner\neg Q \vee D\urcorner=\forall x_{1} . \ldots \forall x_{n} . \neg Q \vee^{-} \delta^{-}|D|$, we can relate the rule $Q \rightarrow^{-} D$ with the clause with selection $\neg Q \vee D$, which is called a one-way clause by Dowek [22]. Indeed, they only differ by the polarity of the delays. However, because of the presence of the $\mathrm{V}^{-}$connective, the delay $\delta^{-}$ is useless in $\ulcorner\neg Q \vee D\urcorner$. Similarly, since the top connective of $|D|$ is $\vee^{+}$, the delay $\delta^{+}$is useless in $\llbracket Q \rightarrow^{-} D \rrbracket$. Ext. Narr. ${ }^{-}$can therefore be seen as an instance of the Resolution with Selection rule:

$$
\text { Resolution with Selection } \frac{\neg Q \vee D \quad P \vee C}{\sigma(D \vee C)} \sigma=m g u(P, Q) \text {. }
$$

Similarly, $P \rightarrow^{+} \neg C$ is related to $\underline{P} \vee C$.

Consequently, since PUSC ${ }^{\perp}$ corresponds to $\mathrm{LKF}^{\perp}$, and Resolution with Input Selection corresponds to Polarized Resolution Modulo theory, Theorem 4.2 leads to a new and more generic proof of the correspondence between PUSC $^{\perp}$ and Polarized Resolution Modulo theory.

Deduction Modulo Theory is not always complete. This is the case only if the cut rule is admissible in Polarized Sequent Calculus Modulo theory. It holds for some particular theories, e.g. Church's Simple Type Theory [23] and arithmetic [25]. There are more or less powerful techniques that ensures this property $[9,21,24,28]$. We even proved that any consistent first-order theory can be presented by a rewriting system admitting the cut rule [8]. As presented with Dowek [11] and discussed in the introduction, the fact that completeness is not proved once for all, but needs to be proved for each particular theory, is essential. Indeed, if a theory is presented entirely by rewriting rules, completeness implies the consistency of the theory, since no rule can be applied on the empty set of clauses. Consequently, the proof of the completeness cannot be easier than the proof of consistency of the theory, and, according to Gödel, cannot be proven in the theory itself.

\subsection{Superdeduction}

We can go a step further than what is done concerning Deduction Modulo Theory and benefit from focusing to decompose the right-hand side formula after an unfolding has occurred. This leads to what Brauner, Houtmann, and Kirchner [5] called Superdeduction. Houtmann [30] studied the links between Superdeduction and focusing, but not with the idea that the rules themselves should be considered as polarized formulas. To link Superdeduction with $\mathrm{LKF}^{\perp}$, we just need to change the translation of rewriting rules in order to ensure that the right-hand side is decomposed as much as possible. This is done by suppressing the positive delay $\delta^{+}$and trying to stay in synchronous (i.e. focused) phase by using negative connectives, until we reach a $\exists$ quantifier, after which we try to 
stay in the asynchronous phase. Note, however, that literals are always given a positive polarization. We introduce the negative translation of a formula:

$$
\begin{aligned}
& \lceil L \mathrm{l}=L \quad \text { when } L \text { is } \mathrm{\top}, \perp \text { or a literal }
\end{aligned}
$$

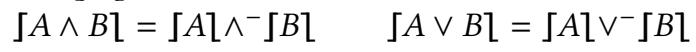

$$
\begin{aligned}
& \lceil\exists x . A \mathrm{l}=\exists x \cdot|A| \quad\lceil\forall x . A \mathrm{l}=\forall x . \varsigma A \mathrm{l}
\end{aligned}
$$

and the translation of rewrite rules becomes:

$$
\begin{aligned}
& \left.\| P \rightarrow^{-} A \rrbracket=\overline{\forall x .} \underline{\neg P} \vee^{-}\right\rfloor A \rrbracket \\
& \left.\rrbracket P \rightarrow^{+} A \rrbracket=\overline{\forall x} . \underline{P} \vee^{-}\right\rfloor A^{\perp} l
\end{aligned}
$$

The synthetic rules given by the translation of rewriting rules correspond exactly to the superrules of Superdeduction.

Example 5.7. Let us consider the proposition rewrite rules defining the natural numbers as the set of terms verifying the inductive predicates, as in [5].

$$
\begin{gathered}
n \in \mathbb{N} \rightarrow \forall p .0 \in p \Rightarrow H(p) \Rightarrow n \in p \\
H(p) \rightarrow \forall m . m \in p \Rightarrow s(m) \in p
\end{gathered}
$$

Here, $p$ is a term representing a class of natural number, as presented in [25]. Each rule is considered with both positive and negative polarities.

The translation above gives the following formulas with polarization:

$$
\begin{gathered}
\forall n . \neg n \in \mathbb{N} \vee^{-}\left(\forall p . \neg 0 \in p \vee^{-} \neg H(p) \vee^{-} n \in p\right) \\
\forall n . \underline{n \in \mathbb{N}} \vee^{-}\left(\exists p .0 \in p \wedge^{+} H(p) \wedge^{+} \neg n \in p\right) \\
\forall p . \neg \underline{H(p)} \vee^{-}\left(\forall m . \neg m \in p \vee^{-} s(m) \in p\right) \\
\forall p . \underline{H(p)} \vee^{-}\left(\exists m . m \in p \wedge^{+} s(m) \in p\right)
\end{gathered}
$$

This leads to the following synthetic rules:

$$
\begin{gathered}
(11) \vdash \frac{\Gamma, n \in \mathbb{N}, \neg 0 \in t \Uparrow \vdash \quad \Gamma, n \in \mathbb{N}, \neg H(t) \Uparrow \vdash \quad \Gamma, n \in \mathbb{N}, n \in t \Uparrow \vdash}{\Gamma, n \in \mathbb{N} \Uparrow \vdash} \\
(12) \vdash \frac{\Gamma, \neg n \in \mathbb{N}, 0 \in p, H(p), \neg n \in p \Uparrow \vdash}{\Gamma, \neg n \in \mathbb{N} \Uparrow \vdash} p \text { not free in } \Gamma \\
(13) \vdash \frac{\Gamma, H(p), \neg u \in p \Uparrow \vdash \quad \Gamma, H(p), s(u) \in p \Uparrow \vdash}{\Gamma, H(p) \Uparrow \vdash} \\
(14) \vdash \frac{\Gamma, \neg H(p), m \in p, \neg s(m) \in p \Uparrow \vdash}{\Gamma, \neg H(p) \Uparrow \vdash} m \text { not free in } \Gamma
\end{gathered}
$$

For instance (12) $\vdash$ corresponds to the following focused derivation: 


$$
\begin{aligned}
& \text { Store } \frac{\Delta, \neg n \in \mathbb{N}, 0 \in p, H(p), \neg n \in p \Uparrow \vdash}{\overline{\Delta, \neg n \in \mathbb{N}, \Uparrow 0 \in p, H(p), \neg n \in p \vdash}} \\
& \Uparrow \exists r \frac{\overline{\Delta, \neg n \in \mathbb{N} \Uparrow 0 \in p \wedge^{+} H(p) \wedge^{+} \neg n \in p \vdash}}{\Delta, \neg n \in \mathbb{N} \Uparrow \exists p .0 \in p \wedge^{+} H(p) \wedge^{+} \neg n \in p \vdash}
\end{aligned}
$$

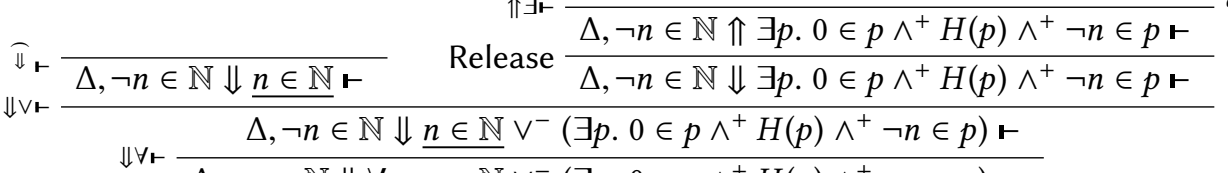

$$
\begin{aligned}
& \text { Focus } \frac{\Delta, \neg n \in \mathbb{N} \Downarrow \forall n . \underline{n \in \mathbb{N}} \vee^{-}\left(\exists p .0 \in p \wedge^{+} H(p) \wedge^{+} \neg n \in p\right) \vdash}{\Delta, \neg n \in \mathbb{N} \Uparrow \vdash}
\end{aligned}
$$

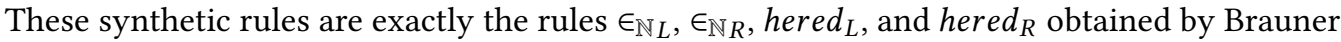
et al. [5], except that in our presentation all formulas are put on the left of the sequent.

Note that the same kind of encodings can be used to show that Definitional reflection, as defined by Schroeder-Heister [35], can be seen as an instance of $\mathrm{LKF}^{\perp}$.

\subsection{Beyond Deduction Modulo Theory}

Example 5.8. Let us recall the set of clauses from the Introduction:

$$
\begin{aligned}
& \neg X \in \mathcal{P}(Y) \vee \neg Z \in X \vee Z \in Y \quad(1) \quad \underline{X \in \mathcal{P}(Y)} \vee d(X, Y) \in X \\
& X \in \mathcal{P}(Y) \vee \neg d(X, Y) \in Y
\end{aligned}
$$

Note that this example is not covered by Ordered Resolution with Selection, at least not if a simplification ordering is used, because we cannot have $X \in \mathcal{P}(Y)>d(X, Y) \in X$ since with $\theta=\{X \mapsto \mathcal{P}(Z) ; Y \mapsto Z\}$ their instances are ordered in the wrong direction: $\mathcal{P}(Z) \in \mathcal{P}(Z) \prec$ $d(\mathcal{P}(Z), Z) \in \mathcal{P}(Z)$.

The synthetic rules of the example from the Introduction correspond to the derivations when one of the clauses is focused on. For instance, if we consider the clause (1), in a context $\Gamma$ containing this clause, a proof putting the focus on $\ulcorner(1)\urcorner$ is necessarily of the following shape:

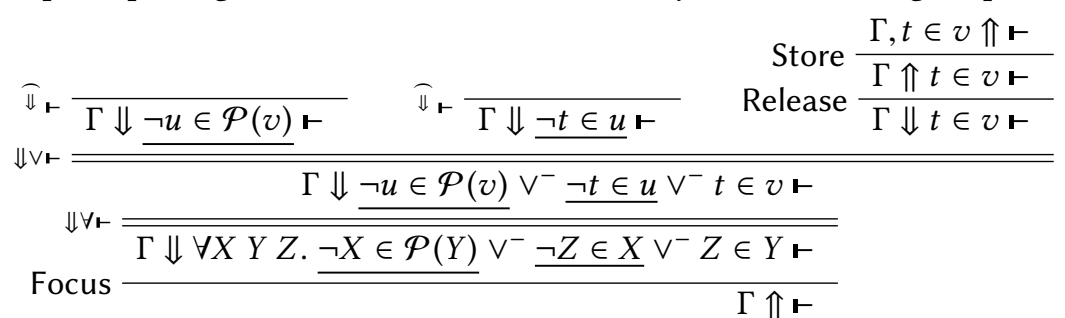

where $t, u, v$ are arbitrary terms, and where, to be able to close the left and middle branches, $u \in \mathcal{P}(v)$ and $t \in u$ must belong to $\Gamma$.

So $\Gamma$ is in fact of the form $\forall X Y Z . \neg X \in \mathcal{P}(Y) \vee^{+} \neg Z \in X \vee^{+} Z \in Y, \Delta, u \in \mathcal{P}(v), t \in u$ for some $\Delta$, and the axiom $\forall X Y Z . \neg X \in \mathcal{P}\left(\overline{Y) \vee^{+} \neg Z \in X} \vee^{+} Z \in Y\right.$ can be replaced by the synthetic rule:

$$
\text { (1) } \frac{\Delta, u \in \mathcal{P}(v), t \in u, t \in v \Uparrow \vdash}{\Delta, u \in \mathcal{P}(v), t \in u \Uparrow \vdash} \text {. }
$$

The computation of the other synthetic rules is left as an exercise for the reader.

Note, by analogy of this synthetic rule with the unfolding rules in $\mathrm{PUSC}^{\perp}$ (see Section 5.2), how (1) can be seen as a rewriting rule that rewrites simultaneously $x \in \mathcal{P}(y)$ and $z \in x$ into $z \in y$. 
On the resolution side, if we consider the ground instances of Resolution with Selection, we have:

$$
\begin{gathered}
\text { R.w.S. } \frac{\neg X \in \mathcal{P}(Y) \vee \neg Z \in X \vee Z \in Y \quad u \in \mathcal{P}(v) \vee C \quad t \in u \vee D}{t \in v \vee C \vee D} \\
\text { R.w.S. } \frac{X \in \mathcal{P}(Y) \vee d(X, Y) \in X \quad \neg u \in \mathcal{P}(v) \vee C}{d(u, v) \in u \vee C} \\
\text { R.w.S. } \frac{X \in \mathcal{P}(Y) \vee \neg d(X, Y) \in Y}{C \vee u \in \mathcal{P}(v) \vee C \quad d(u, v) \in v \vee D}
\end{gathered}
$$

hence the derived rules given in the introduction, where the clauses of the theory are not mentioned.

The question that remains is how we can prove the completeness of such a selection. We consider the case when there is a fixed selection on a finite set of clauses, and arbitrary other clauses without selection. The intuition is that the clauses with selection constitute the theory in which the proof is searched for, whereas the other clauses represent some goal to be proven in that theory. In that case, one can consider the second projection $\mathcal{S}$ attached to the clauses of the theory, which we call a selection.

We can in fact consider only subselections.

Definition 5.9 (Singleton subselection). Given a selection $\mathcal{S}$, the selection $\mathcal{S}_{1}$ is a singleton subselection of $\mathcal{S}$ if

- $\mathcal{S}_{1}(C) \subseteq \mathcal{S}(C)$ for all $C \in \Gamma$

- if $\mathcal{S}(C) \neq \emptyset$ then $\operatorname{card}\left(\mathcal{S}_{1}(C)\right)=1$.

Example 5.10. A singleton subselection of Example 5.8 can be

$\neg X \in \mathcal{P}(Y) \vee \underline{\neg Z \in X} \vee Z \in Y \quad \underline{X \in \mathcal{P}(Y)} \vee d(X, Y) \in X \quad \underline{X \in \mathcal{P}(Y)} \vee \neg d(X, Y) \in Y$

In the case of propositional logic, it is enough to consider only singleton subselections. To show this, given a selection $\mathcal{S}$, let us arbitrarily order the occurrences of literals selected by $\mathcal{S}$ in each clause of the theory. Singleton subselections can be partially ordered by the transitive closure of the following relation : $\mathcal{S}_{1}$ is greater than $\mathcal{S}_{1}^{\prime}$ if in some clause, $\mathcal{S}_{1}$ selects a greater literal than $\mathcal{S}_{1}^{\prime}$. Since there are only finitely many subselections, this ordering is well-founded.

We prove the following lemma:

Lemma 5.11. Given a selection $\mathcal{S}$ and a singleton subselection $\mathcal{S}_{1}$, assume that for all literals $L$ that are selected by $\mathcal{S}$ and that are greater than the literal selected by $\mathcal{S}_{1}, L^{\perp}$ is in $\Gamma$.

If for all singleton subselections $\mathcal{S}_{1}^{\prime}$ smaller than or equal to $\mathcal{S}_{1}$, the sequent $\ulcorner\Gamma\urcorner \Uparrow \vdash$ has a proof using $\mathcal{S}_{1}^{\prime}$, then this sequent has a proof using $\mathcal{S}$.

Proof. By lexicographic induction on $\mathcal{S}_{1}$ and on the proof of $\ulcorner\Gamma\urcorner \Uparrow \vdash$ using it.

If the last proof step is valid using $\mathcal{S}$, we apply it and proceed by induction on the subproofs.

If it is not, this means that the negation of some literal that is not selected in $\mathcal{S}_{1}$ but that is selected in $\mathcal{S}$ is not in the context $\ulcorner\Gamma\urcorner$, thus preventing to close a branch using $\mathbb{\Downarrow}$. In other words, the proof ends by focusing on a formula corresponding to a clause $K_{1} \vee \cdots \vee K_{n} \vee C$ where the $K_{i} \mathrm{~s}$ are in decreasing order, $\mathcal{S}\left(K_{1} \vee \ldots \vee K_{n} \vee C\right)=\left\{K_{1}, \ldots, K_{n}\right\}$ and $\mathcal{S}_{1}\left(K_{1} \vee \ldots \vee K_{n} \vee C\right)=\left\{K_{j}\right\}$, and we have a derivation of the form: 


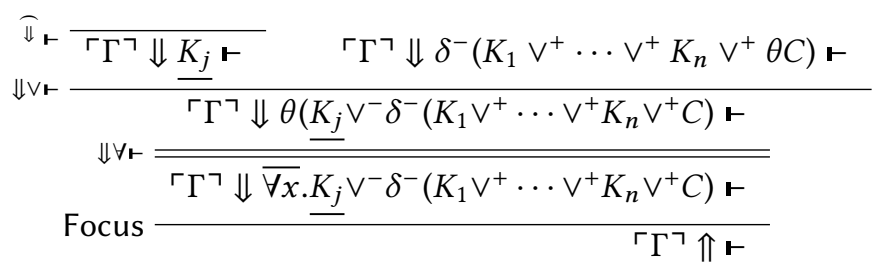

using $\mathcal{S}_{1}$.

By assumption, all $K_{i}{ }^{\perp}$ for $i<j$ are in $\ulcorner\Gamma\urcorner$. To close the left branch, we also have $K_{j}{ }^{\perp}$ in $\ulcorner\Gamma\urcorner$. Let us now consider the singleton subselection equal to $\mathcal{S}_{1}$ except that it selects $K_{j+1}$ instead of $K_{j}$ in $K_{1} \vee \cdots \vee K_{n} \vee C$. This subselection is smaller than $\mathcal{S}_{1}$, so we can apply the induction hypothesis to obtain a proof using $\mathcal{S}$.

TheOREM 5.12. Assume that $\mathcal{S}$ selects only ground literals.

Resolution with input selection $\mathcal{S}$ is complete iff for all singleton subselections $\mathcal{S}_{1}$ of $\mathcal{S}$, Resolution with input selection $\mathcal{S}_{1}$ is complete.

Proof. For the "only if" part, just note that a Resolution with Selection step using $\mathcal{S}$ can easily be simulated by a Resolution with Selection step using $\mathcal{S}_{1}$ plus possibly one or several applications of Resolution.

Assume $\Gamma$ to be unsatisfiable. Since Resolution with input selection is complete for all singleton subselection $\mathcal{S}_{1}$, it means that for each singleton subselection $\mathcal{S}_{1}$ the sequent $\ulcorner\Gamma\urcorner \Uparrow \vdash$ is provable using $\mathcal{S}_{1}$, according to Theorem 4.12. Let us consider $\mathcal{S}_{1}$ the singleton subselection selecting the greatest selected literal in each clause, if it exists. The hypothesis of Lemma 5.11 are fulfilled, hence $\ulcorner\Gamma\urcorner \Uparrow \vdash$ has a proof using $\mathcal{S}$. Using Theorem 4.7, the empty clause can be derived from $\Gamma$ in Resolution with input selection $\mathcal{S}$. It is therefore complete.

We conjecture that the same theorem holds even if non-ground literals are selected.

Since singleton subselections can be linked with rewriting systems in Deduction Modulo Theory according to Section 5.2, we could reduce the problem of completeness in our framework to several problems of completeness in Deduction Modulo Theory.

In particular, to prove completeness if we have a clause $L_{1} \vee \ldots \vee L_{n} \vee K_{1} \vee \ldots \vee K_{m}$, one could prove completeness of the $n$ singleton subselections

$$
\begin{gathered}
\underline{L_{1} \vee \ldots \vee L_{n} \vee K_{1} \vee \ldots \vee K_{m}} \\
\vdots \\
L_{1} \vee \ldots \vee \underline{L_{n}} \vee K_{1} \vee \ldots \vee K_{m}
\end{gathered}
$$

which correspond, according to Section 5.2, to the $n$ rewriting systems

$$
\begin{aligned}
& L_{1} \rightarrow^{ \pm}(\neg) \overline{\forall x} . L_{2} \vee \cdots \vee L_{n} \vee K_{1} \vee \cdots \vee K_{m} \\
& \vdots \\
& L_{n} \rightarrow^{ \pm}(\neg) \overline{\forall x .} L_{1} \vee \cdots \vee L_{n-1} \vee K_{1} \vee \cdots \vee K_{m}
\end{aligned}
$$

in Deduction Modulo Theory. 
Example 5.13. Following Example 5.8, proving the completeness of our selection would amount to proving cut admissibility in Deduction Modulo Theory for the four following rewriting systems:

$$
\begin{gathered}
X \in \mathcal{P}(Y) \rightarrow^{-} \forall Z . \neg Z \in X \vee Z \in Y \\
X \in \mathcal{P}(Y) \rightarrow^{+} \neg d(X, Y) \in X \\
X \in \mathcal{P}(Y) \rightarrow^{+} d(X, Y) \in Y \\
Z \in X \rightarrow^{-} \forall Y . \neg X \in \mathcal{P}(Y) \vee Z \in Y \\
X \in \mathcal{P}(Y) \rightarrow^{+} \neg d(X, Y) \in X \\
X \in \mathcal{P}(Y) \rightarrow^{+} d(X, Y) \in Y \\
X \in \mathcal{P}(Y) \rightarrow^{-} \forall Z . \neg Z \in X \vee Z \in Y \\
X \in \mathcal{P}(Y) \rightarrow^{+} \neg d(X, Y) \in X \\
d(X, Y) \in Y \rightarrow^{-} X \in \mathcal{P}(Y) \\
Z \in X \rightarrow^{-} \forall Y . \neg X \in \mathcal{P}(Y) \vee Z \in Y \\
X \in \mathcal{P}(Y) \rightarrow^{+} \neg d(X, Y) \in X \\
d(X, Y) \in Y \rightarrow^{-} X \in \mathcal{P}(Y)
\end{gathered}
$$

Cut admissibility of these systems can be proved by analyzing so-called critical proofs as defined by Burel and Kirchner [12].

\section{CONCLUSION AND FURTHER WORK}

We generalized focusing and resolution with selection, proved that they correspond, and showed how known calculi are instances of this framework, namely ordinary focusing, hyperresolution, Deduction Modulo Theory and Superdeduction. We also showed how to reduce completeness of this framework to several completeness proofs in Deduction Modulo Theory. We can therefore reuse the various techniques for proving completeness in Deduction Modulo Theory [9, 21, 24, 28] in our framework. As Deduction Modulo Theory already gives significant results in industrial applications when the theory is a variant of set theory (more precisely, set theory of the B method) [13], we can expect our framework to lead to even better outcomes. The notable results presented here raise the following new areas of investigations.

First, we need to study how to apply selection also in the generated clauses. This should allow us to cover the cases of Ordered Resolution with Selection and of Semantic Resolution in the first-order case. Dually, in the sequent calculus part, this would correspond to the possibility to dynamically add selection in formulas of subderivations. This could probably be linked with the work of Deplagne [19] where rewrite rules corresponding to induction hypotheses are dynamically added in the rewriting system of a sequent calculus for Deduction Modulo Theory. Note that we already have one direction, namely from Resolution with Input Selection to $\mathrm{LKF}^{\perp}$, since Lemmas $4.8,4.10$, and 4.11 do not assume anything on the generated clauses; except, for Factoring, that it selects at least the instances of literals that were already selected. The converse direction would require a meta-theorem of completeness, since it is obviously not complete for all possible dynamic choices of selection.

Since focusing is defined not only for classical first-order logic but also for linear, intuitionistic, modal logics, the work in this paper could serve as a starting point to study how to get automated proof-search methods for these logics with a selection mechanism. 
Another worthwhile point is how equality should be handled in our framework. In particular, it would be interesting to see how paramodulation calculi, in particular superposition, can be embedded into a sequent calculus.

Finally, it would be worth investigating whether completeness proofs based on model construction, such as semantic completeness proofs of tableaux (related to sequent calculus), and completeness proof of superposition [2], can be related one to the other in our framework.

\section{ACKNOWLEDGMENTS}

We would like to thank Dale Miller and Kaustuv Chaudhuri for their comments, as well as the anonymous referees for their precious remarks.

\section{REFERENCES}

[1] Jean-Marc Andreoli. 1992. Logic Programming with Focusing Proofs in Linear Logic. fournal of Logic and Computation 2, 3 (1992), 297-347.

[2] L. Bachmair and H. Ganzinger. 1994. Rewrite-based equational theorem proving with selection and simplification. fournal of Logic and Computation 4, 3 (1994), 1-31.

[3] Leo Bachmair and Harald Ganzinger. 2001. Resolution Theorem Proving. In Handbook of Automated Reasoning, John Alan Robinson and Andrei Voronkov (Eds.). Elsevier and MIT Press, 19-99.

[4] Richard Bonichon and Olivier Hermant. 2006. A Semantic Completeness Proof for TaMed. In LPAR (LNCS), Miki Hermann and Andrei Voronkov (Eds.), Vol. 4246. Springer, 167-181.

[5] Paul Brauner, Clément Houtmann, and Claude Kirchner. 2007. Principle of superdeduction. In LICS, Luke Ong (Ed.). 41-50.

[6] Guillaume Burel. 2010. Embedding Deduction Modulo into a Prover. In CSL (LNCS), Anuj Dawar and Helmut Veith (Eds.), Vol. 6247. Springer, 155-169.

[7] Guillaume Burel. 2011. Experimenting with Deduction Modulo. In CADE (LNCS), Viorica Sofronie-Stokkermans and Nikolaj Bjørner (Eds.), Vol. 6803. Springer, 162-176.

[8] Guillaume Burel. 2013. From Axioms to Rewriting Rules. (2013). Available at http://web4.ensiie.fr/ guillaume.burel/ download/burel13axioms.pdf.

[9] Guillaume Burel. 2014. Cut Admissibility by Saturation. In RTA-TLCA (LNCS), Gilles Dowek (Ed.), Vol. 8560. Springer, $124-138$.

[10] Guillaume Burel. 2018. Linking Focusing and Resolution with Selection. In MFCS 2018 (LIPIcs), Igor Potapov, Paul Spirakis, and James Worrell (Eds.), Vol. 117. Schloss Dagstuhl-Leibniz-Zentrum fãijr Informatik, Dagstuhl, Germany, 9:1-9:14. https://doi.org/10.4230/LIPIcs.MFCS.2018.9

[11] Guillaume Burel and Gilles Dowek. 2009. How can we prove that a proof search method is not an instance of another?. In LFMTP (ACM International Conference Proceeding Series). ACM, 84-87.

[12] Guillaume Burel and Claude Kirchner. 2010. Regaining Cut Admissibility in Deduction Modulo using Abstract Completion. Information and Computation 208, 2 (2010), 140-164.

[13] Guillaume Bury, David Delahaye, Damien Doligez, Pierre Halmagrand, and Olivier Hermant. 2015. Automated Deduction in the B Set Theory using Typed Proof Search and Deduction Modulo. In LPAR (EPiC Series in Computing), Ansgar Fehnker, Annabelle McIver, Geoff Sutcliffe, and Andrei Voronkov (Eds.), Vol. 35. EasyChair, 42-58. http: //www.easychair.org/publications/volume/LPAR-20

[14] Samuel R. Buss (Ed.). 1998. Handbook of proof theory. Elsevier, Amsterdam.

[15] Kaustuv Chaudhuri and Frank Pfenning. 2005. A Focusing Inverse Method Theorem Prover for First-Order Linear Logic. In CADE (LNCS), Robert Nieuwenhuis (Ed.), Vol. 3632. Springer, 69-83.

[16] Kaustuv Chaudhuri, Frank Pfenning, and Greg Price. 2008. A Logical Characterization of Forward and Backward Chaining in the Inverse Method. Journal of Automated Reasoning 40, 2-3 (2008), 133-177.

[17] Zakaria Chihani, Tomer Libal, and Giselle Reis. 2015. The Proof Certifier Checkers. In TABLEAUX (LNCS), Vol. 9323. Springer, Wroclaw, Poland, 201-210.

[18] Zakaria Chihani, Dale Miller, and Fabien Renaud. 2013. Foundational Proof Certificates in First-Order Logic. In $C A D E$ (LNCS), Maria Paola Bonacina (Ed.), Vol. 7898. Springer, 162-177.

[19] Eric Deplagne and Claude Kirchner. 2004. Induction as Deduction Modulo. Rapport de recherche. LORIA. http: //www.loria.fr/publications/2004/A04-R-468/A04-R-468.ps

[20] Gilles Dowek. 2002. What Is a Theory?. In STACS (LNCS), Helmut Alt and Afonso Ferreira (Eds.), Vol. 2285. Springer, 50-64. 
[21] Gilles Dowek. 2006. Truth Values Algebras and Proof Normalization. In TYPES (LNCS), Thorsten Altenkirch and Conor McBride (Eds.), Vol. 4502. Springer, 110-124.

[22] Gilles Dowek. 2010. Polarized Resolution Modulo. In IFIP TCS (IFIP AICT), Cristian S. Calude and Vladimiro Sassone (Eds.), Vol. 323. Springer, 182-196.

[23] Gilles Dowek, Thérèse Hardin, and Claude Kirchner. 2003. Theorem Proving Modulo. fournal of Automated Reasoning 31, 1 (2003), 33-72.

[24] Gilles Dowek and Benjamin Werner. 2003. Proof Normalization Modulo. The fournal of Symbolic Logic 68, 4 (2003), 1289-1316.

[25] Gilles Dowek and Benjamin Werner. 2005. Arithmetic as a Theory Modulo. In RTA (LNCS), Jürgen Giesl (Ed.), Vol. 3467. Springer, 423-437.

[26] Mahfuza Farooque, Stéphane Graham-Lengrand, and Assia Mahboubi. 2013. A bisimulation between DPLL(T) and a proof-search strategy for the focused sequent calculus. In LFMTP, Alberto Momigliano, Brigitte Pientka, and Randy Pollack (Eds.). ACM, 3-14

[27] Jean Goubault-Larrecq. 2002. A Note on the Completeness of Certain Refinements of Resolution. Research Report LSV-02-8. Laboratoire Spécification et Vérification, ENS Cachan, France.

[28] Olivier Hermant. 2005. Méthodes Sémantiques en Déduction Modulo. Ph.D. Dissertation. École Polytechnique.

[29] Olivier Hermant. 2009. Resolution is Cut-Free. fournal of Automated Reasoning 44, 3 (2009), 245-276.

[30] Clément Houtmann. 2008. Axiom directed Focusing. In Types for Proofs and Programs (LNCS), Stephano Berardi, Ferruccio Damiani, and Ugo de'Liguoro (Eds.), Vol. 5497. Springer, 169-185.

[31] Chuck Liang and Dale Miller. 2009. Focusing and polarization in linear, intuitionistic, and classical logics. Theoretical Computer Science 410, 46 (2009), 4747-4768. Abstract Interpretation and Logic Programming: In honor of professor Giorgio Levi.

[32] Sean McLaughlin and Frank Pfenning. 2008. Imogen: Focusing the Polarized Inverse Method for Intuitionistic Propositional Logic. In LPAR (Lecture Notes in Computer Science), Iliano Cervesato, Helmut Veith, and Andrei Voronkov (Eds.), Vol. 5330. Springer, 174-181.

[33] Dale Miller and Marco Volpe. 2015. Focused Labeled Proof Systems for Modal Logic. In LPAR (LNCS), Martin Davis, Ansgar Fehnker, Annabelle McIver, and Andrei Voronkov (Eds.), Vol. 9450. Springer, 266-280.

[34] J. A. Robinson. 1965. Automatic Deduction with Hyper-Resolution. International fournal of Computer Mathematics 1 (1965), 227-234.

[35] Peter Schroeder-Heister. 1990. Cut Elimination for Logics with Definitional Reflection.. In Nonclassical Logics and Information Processing (LNCS), Vol. 619. Springer, 146-171.

[36] James R. Slagle. 1967. Automatic Theorem Proving With Renamable and Semantic Resolution. f. ACM 14, 4 (1967), 687-697.

[37] Larry Wos, George A. Robinson, and Daniel F. Carson. 1965. Efficiency and Completeness of the Set of Support Strategy in Theorem Proving. J. ACM 12, 4 (1965), 536-541. 